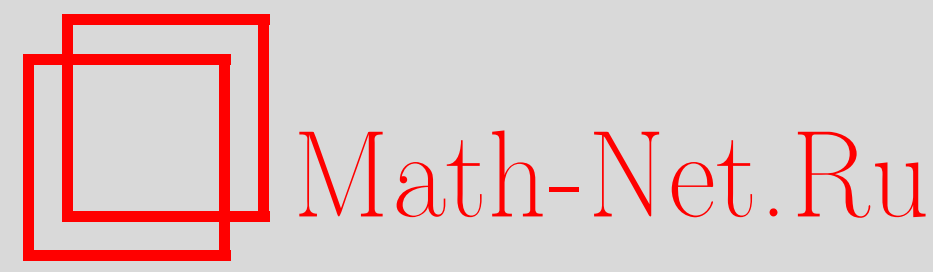

В. Г. Кановей, М. Реекен, Некоторые новые результаты о борелевской несводимости отношений эквивалентности, Изв. РАН. Сер. матем., 2003, том 67, выпуск $1,59-82$

DOI: https://doi.org/10.4213/im418

Использование Общероссийского математического портала Math-Net.Ru подразумевает, что вы прочитали и согласны с пользовательским соглашением

http://www . mathnet.ru/rus/agreement

Параметры загрузки:

IP : 54.198 .187 .58

26 апреля 2023 г., 17:54:44 
УДК 510.225

\author{
В. Г. Кановей, М. Реекен
}

\title{
Некоторые новые результаты о борелевской несводимости отношений эквивалентности
}

\begin{abstract}
Доказано, что отношения эквивалентности (кратко, ОЭ), индуцированные генерически турбулентными "польскими" действиями, борелевски несводимы к ОЭ из семейства, включающего "польские" действия группы $S_{\infty}$ всех перестановок $\mathbb{N}$ и замкнутого относительно произведений Фубини по модулю идеала Fin конечных множеств и некоторых других операций. Показано, что $\mathrm{T}_{2}$ - отношение эквивалентности, называемое равенством счетных множеств вещественных чисел, - борелевски несводимо к другому семейству ОЭ, которое включает непрерывные действия "польских" CLI-групп, борелевские отношения эквивалентности с $\mathbf{G}_{\delta \sigma}$-классами, некоторые идеалы и замкнуто относительно произведений Фубини по модулю Fin. Оба эти результата и их следствия усиливают некоторые более ранние теоремы несводимости Хъёрта и Кехриса.

Библиография: 15 наименований.
\end{abstract}

\section{Введение}

Классификационные проблемы для разных типов математических структур были в центре внимания дескриптивной теории множеств в последние 10-15 лет. Предположим, что $X$ - класс математических структур, идентифищированных по модулю отношения эквивалентности Е. Это могут быть, например, счетные группы с точностью до изоморфизма, унитарные операторы над фиксированным пространством $\mathbb{C}^{n}$ с точностью до конъюгации, вероятностные меры на фиксированном "польском" пространстве с точностью до отождествления мер, имеющих одни и те же множества меры 0 или, скажем, вещественные числа по модулю тьюринговой эквивалентности. (Примеры взяты из книги [6] и статьи [12], где приведены многие другие примеры.) Допустим, что $Y$ - другой класс математических структур, идентифицированных с точностью до отношения эквивалентности $F$. Тогда классификационная проблема состоит в том, чтобы выяснить, существует ли определимая, или әффективная, инъекция $\Theta: X / \mathrm{E} \rightarrow Y / \mathrm{F}$. Такое отображение $\Theta$ может рассматриваться как классификация объектов класса $X$ в терминах объектов из $Y$, согласованная с факторизацией по $\mathrm{E}$ и F. Существование этого отображения может являться важным результатом, например, когда объекты из $Y$ имеют более простую математическую природу, чем объекты из $X$.

Результаты этой статьи были частично представлены на конференциях LC'1999 (Утрехт), LC'2000 (Париж), а также на конференции по теории множеств Lumini-2000 и семинарах Калтеха и UCLA и конференции памяти П. С. Новикова (Москва, 2001).

Работа первого автора выполнена при поддержке гранта DFG 101/10-1.

(C) В.Г. КАновей, М. Реeкен, 2003 
Во многих случаях оказывается, что классы структур $X$ и $Y$ могут рассматриваться как “польские" (т. е. сепарабельные полные метрические) пространства, и при этом E, F становятся борелевскими или, более общо, аналитическими (как множества пар) отношениями, а на отображения обычно накладывается требование быть борелевскими ${ }^{1}$. В этом случае проблема может изучаться методами дескриптивной теории множеств, где она принимает следуюшую форму: если $\mathrm{E}, \mathrm{F}$ борелевские или аналитические отношения эквивалентности на "польских" пространствах $X, Y$ соответственно, то возникает вопрос: сушествует ли борелевская редукиия $\mathrm{E}$ к $\mathrm{F}$, т. е. борелевское отображение $\vartheta: X \rightarrow Y$, удовлетворяющее соотношению $x \mathrm{E} x^{\prime} \Longleftrightarrow \vartheta(x) \mathrm{F} \vartheta\left(x^{\prime}\right)$ для всех $x, x^{\prime} \in X$ ? Если такое отображение $\vartheta$ сушествует, то говорят, что $\mathrm{E}$ борелевски сводимо к $\mathrm{F}$. При изучении борелевских и аналитических отношений эквивалентности (кратко: ОЭ) в терминах борелевской сводимости методами дескриптивной теории множеств была вскрыта замечательная структура теорем сводимости и несводимости между ОЭ разных типов (см. обзоры полученных результатов в [2], [5]-[7], [12]). Настояшая статья принадлежит к этому исследовательскому направлению.

Основная теорема 1 доказывает борелевскую несводимость одного большого класса ОЭ к другому. Класс 1 состоит из ОЭ, индуцированных генерически турбулентнылми 2 "польскими" действиями. Г. Хъёрт [6] доказал, что ОЭ этого класса борелевски не сводятся к ОЭ класса 2, состояшего из ОЭ, индуцированных “польскими" действиями $S_{\infty}-$ группы всех перестановок $\mathbb{N}$. (Этот результат также известен в следующей формулировке: генерически турбулентные ОЭ не классифицируемы счетными структурами; см. п.3.3.)

Одно из возможных доказательств теоремы Хъёрта состоит в следующем. Во-первых, любое ОЭ, борелевски сводимое к $\mathrm{O}$ эласса 2, также борелевски сводимо по крайней мере на котощем (comeager) множестве (т.е. дополнительном к множеству первой категории) к отношению класса $3^{3}$, состояшего из всех ОЭ, которые можно получить из отношений равенства на "польских" пространствах при помощи итераций операции счетной степени $\mathrm{E}^{\infty}$. Во-вторых (здесь применяется теория турбулентности Хъёрта), ОЭ класса 1 борелевски несводимы к ОЭ класса 3 даже на котощих множествах. Теорема 1 обобщает вторую часть. Рассматривается класс 4, содержаший те ОЭ, которые получаются из равенств на "польских" пространствах операциями:

1) счетного объединения (если оно дает ОЭ) данных ОЭ на одном и том же пространстве;

2) произведения Фубини $\prod_{k \in \mathbb{N}} \mathrm{E}_{k} /$ Fin по модулю идеала Fin всех конечных подмножеств $\mathbb{N}$;

3) счетной степени $\mathrm{E}^{\infty}$

(см. точные определения в п. 1.2).

\footnotetext{
${ }^{1}$ Т. е. с борелевскими графами. Также рассматриваются отображения, измеримые по Бэру, и редукции, удовлетворяющие некоторым алгебраическим требованиям [2], а также $\Delta_{2}^{1}$ и более сложные редукции [9], [10], однако они не будут рассматриваться в настоящей статье.

${ }^{2}$ Т.е. все, с точностью до первой категории, орбиты и даже локальные орбиты должны быть где-то плотными (см. определение 5).

${ }^{3}$ Введенного, по существу, Х. Фридманом [3], [4].
} 
Класс 4, разумеется, включает класс 3 , но содержит значительно больше различных $\mathrm{O} Э$, в частности, определяемых при помощи произведения Фубини, например все ОЭ, порождаемые обобщенными идеалами Фреше, неразложимыми идеалами и идеалами Вейсса (см. п. 1.2).

ТЕОРема 1. ОЭ класса 1 борелевски несводимы (даже несводимы функииями, измеримыми по Бәру) кОЭ класса 4.

Доказательство (см. §2) использует индукцию по построению ОЭ класса 4 с помощью указанных операций. Доказательство основано на технике теории турбулентности, в частности, ключевым шагом будет проверка того, что ОЭ класса 1 являются генерически эргодическими по отношению к ОЭ класса 4 (теорема 6). Приложением этого результата будет доказательство вьшеупомянутой теоремы Хъёрта в результате нескольких достаточно простых рассуждений, проведенных в $\S 3$.

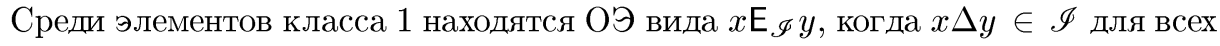
$x, y \in \mathscr{P}(\mathbb{N})$, где $\mathscr{I}$ - идеал на $\mathbb{N}$. Любой идеал $\mathscr{I} \subseteq \mathscr{P}(\mathbb{N})$ является, очевидно, абелевой группой с симметрической разностью $\Delta$ в качестве групповой операции, а $\mathrm{E}_{\mathscr{I}}$ индуцируется сдвиговым действием $\mathscr{I}$ на $\mathscr{P}(\mathbb{N})$ посредством $\Delta$. A. Kexрис [11] показал, что это действие турбулентно в случае, когда $\mathscr{I}$ - борелевский $P$-идеал ${ }^{4}$ с некоторыми исключениями, указанньми ниже. Это позволяет доказать в п. 3.1 следуюшую теорему как следствие теоремы 1.

Теорема 2. Если $\mathscr{Z}$ - нетривиальный ${ }^{5}$ борелевский $P$-идеал на $\mathbb{N}$, mо $\mathrm{E}_{\mathscr{Z}}$ борелевски несводимо $к$ ОЭ класса 4 , кроме случаев, когда идеал $\mathscr{Z}$ либо является идеалом конечных множеств Fin, либо тривиальной вариацией Fin, либо изоморфен идеалу $\mathscr{I}_{3}=0 \times$ Fin через биекиил между базовыми множествами.

Борелевские $P$-идеалы образуют интенсивно изучаемый класс, включающий, например, Fin, идеал $\mathscr{I}_{3}=0 \times$ Fin всех множеств $x \subseteq \mathbb{N}^{2}$ таких, что каждое сечение $(x)_{n}=\{k:\langle n, k\rangle \in x\}$ конечно, и тривиальные вариации Fin, т. е. идеалы вида $\{x \in \mathscr{P}(\mathbb{N}): x \cap W \in \mathrm{Fin}\}$, где $W \subseteq \mathbb{N}$ бесконечно (см. п. 1.3), а также суммируемые идеалы, идеалы плотности и многие другие (см. [2], [15]). Легко видеть, что ОЭ $\mathrm{E}_{0}=\mathrm{E}_{\mathrm{Fin}}$ и $\mathrm{E}_{3}=\mathrm{E}_{\mathscr{I}_{3}}$, порождаемые идеалами Fin и $\mathscr{I}_{3}$, и те, которые соответствуют тривиальным вариациям Fin, принадлежат классу 4, так что исключение Fin, $\mathscr{I}_{3}$ и тривиальных вариаций Fin в теореме 2 необходимо и полностью мотивировано.

Отметим, что более слабая форма теоремы 2 , с классом 3 вместо класса 4 , по сушеству доказана А. Кехрисом [11]. Частньй результат о том, что ОЭ $\mathrm{E}_{\mathscr{Z}_{0}}$, индуцированное идеалом нулевой плотности $\mathscr{Z}_{0}$, борелевски несводимо к любому ОЭ класса 3 , объявлен в [3] (доказательство см. в [4]).

В заключительном $\S 4$ сделана попытка получить результаты обратного характера, т. е. что ОЭ класса 4 борелевски несводимы, скажем, к турбулентным или иным ОЭ другой природы. Это сравнительно менее развитая область, и известна,

\footnotetext{
${ }^{4}$ Идеал $\mathscr{I}$ на $\mathbb{N}$ называется $P$-идеалом, если для любой последовательности множеств $x_{n} \in \mathscr{I}$ имеется множество $x \in \mathscr{I}$ такое, что $x_{n} \backslash x$ конечно для всех $n$.

${ }^{5}$ Содержащий все одноэлементные множества $\{n\}, n \in \mathbb{N}$, и не равный $\mathscr{P}(\mathbb{N})$.
} 
пожалуй, только одна теорема такого типа: Хъёрт [5] доказал, что ОЭ $\mathrm{T}_{2}$ на счетных последовательностях вещественных чисел, определенное как $\left\{x_{n}\right\} \boldsymbol{T}_{2}\left\{y_{n}\right\}$, если $\left\{x_{n}: n \in \mathbb{N}\right\}=\left\{y_{n}: n \in \mathbb{N}\right\}^{6}$, борелевски несводимо ни к какому ОЭ, порожденному непрерьвным действием "польской" группы, допускающей полную инвариантную слева метрику (CLI-груnпа, от термина complete left invariant; это включает, например, "польские" абелевы групшы). Можно ожидать, что Т 2 борелевски не сводится даже к борелевским действиям борелевских абелевых групп, но пока это является открытой проблемой, даже по отношению к сдвиговым $\Delta$-действиям борелевских идеалов.

Возможный путь к разрешению этой проблемы связан со следующим требованием к ОЭ Е (неявно введенным в [5]): для любого форсинга $\mathbb{P} u \mathbb{P}$-терла $\boldsymbol{\xi}$, если $\mathbb{P} \times \mathbb{P}$ вынуждает $\boldsymbol{\xi}_{\text {л }} \mathrm{E} \boldsymbol{\xi}_{\text {пр }}$, имеется точка $x$ в исходном универсуме такая, ито $\mathbb{P}$ вынуждает $x \mathrm{E} \boldsymbol{\xi}$. Мы называем приколотыми все ОЭ Е, удовлетворяющие этому условию. Само $\mathrm{T}_{2}$ не приколото и борелевски не сводится ни к какому аналитическому приколотому ОЭ. Докажем в $\S 4$, что:

1) все ОЭ, индуцированные "польскими" действиями CLI-групп, приколоты (наше доказательство упрошает то доказательство, которое дал Хъёрт в [5]);

2 ) борелевские $\mathrm{O}$ Э, чьи классы эквивалентности принадлежат $\mathbf{G}_{\delta \sigma}$, приколоты (это основано на идее, сообщенной нам Хъёртом);

3) ОЭ, порожденные истощающими идеалами последовательностей субмер на $\mathbb{N}$ (не все из них допускают "польскую" топологию, совместимую с $\Delta$ ), приколоты;

4) произведения Фубини аналитических приколотых ОЭ по модулю Fin приколоты.

Все эти ОЭ борелевски не сводят $\mathrm{T}_{2}$. В частности, все $\mathrm{O} Э$, порожденные идеалами $\Phi$ реше, приколоты и борелевски не сводят $\mathrm{T}_{2}$.

\section{§1. Базовые определения}

Этот параграф содержит обзор основных обозначений, фигурируюших в формулировках и доказательствах теорем 1 и 2.

1.1. Дескриптивная теория множеств. Предполагается некоторое знакомство читателя с теорией борелевских и аналитических множеств в "польских" (т.е. сепарабельных полных метрических) пространствах. Напомним, что аналитические множества (также известные как суслинские, $A$-множества или $\boldsymbol{\Sigma}_{1}^{1}$ ) это непрерывные образы борелевских множеств. Любое борелевское множество является аналитическим, но (в несчетных "польских" пространствах) обратное неверно.

Отображение $f$ (между борелевскими множествами в "польских" пространствах) называется борелевским, если его график - борелевское множество, или, что равносильно, если все $f$-прообразы открытых множеств - борелевские множества. Отображение $f: \mathbb{X} \rightarrow \mathbb{Y}$ является измеримым по Бәру, когда все $f$-прообразы открытых множеств в $\mathbb{Y}$ имеют свойство Бэра в $\mathbb{X}$ (иными словами, совпадают с открытыми множествами с точностью до тощих множеств, т. е. множеств первой ка-

\footnotetext{
${ }^{6} \mathrm{~T}_{2}$ иногда обозначают $\mathrm{F}_{2}[6]$ и часто называют равенством счетных множеств вещественных чисел; оно входит в класс 3 и является одним из наиболее важных борелевских ОЭ.
} 
тегории). Любое такое отображение непрерывно на плотном $\mathbf{G}_{\delta}$-множестве $D \subseteq \mathbb{X}$ (пространства $\mathbb{X}, \mathbb{Y}$ предполагаются “польскими”).

Суперпозиции борелевских отображений, очевидно, сами являются борелевскими. Это, вообще говоря, не верно для измеримых по Бэру отображений, однако имеется полезный частный результат.

Лемма 3. Для “польских" пространств $\mathbb{X}, \mathbb{Y}, \mathbb{Z}$, если отображения $f: \mathbb{X} \rightarrow$ $\mathbb{Y} u g: \mathbb{Y} \rightarrow \mathbb{Z}$ являются соответственно измеримым по Бәру и борелевским, суперпозичия $f \circ g: \mathbb{X} \rightarrow \mathbb{Z}$ измерима по Бәру.

ДокАЗАТЕЛЬСТВО. По определению $g$-прообразы открытых подмножеств $\mathbb{Z}$ являются борелевскими в $\mathbb{Y}$, а их $f$-прообразы - борелевские комбинации множеств со свойством Бэра.

1.2. Отношения эквивалентности и сводимость. Через $\mathrm{D}(X)$ обозначается равенство на $X$, рассматриваемое как $\mathrm{Э}$.

Рассмотрим ОЭ Е на множестве $X$. Тогда $[y]_{\mathrm{E}}=\{x \in X: y \mathrm{E} x\}$ называется Е-классом элемента $y \in X$. Множество $Y \subseteq X$ называется попарно Е-эквивалентнылм, когда $x \mathrm{E} y$ выполнено для всех $x, y \in Y$.

Допустим, что $\mathrm{E}$ и $\mathrm{F}-\mathrm{O}$ Э на "польских" пространствах $\mathbb{X}$ и $\mathbb{Y}$. Введем следующие определения:

(i) $\mathrm{E} \leqslant_{\mathrm{B}} \mathrm{F}$ (борелевская сводимость; иногда пишут $\left.\mathbb{X} / \mathrm{E} \leqslant_{\mathrm{B}} \mathbb{Y} / \mathrm{F}\right)$ означает, что существует борелевское отображение $\vartheta: \mathbb{X} \rightarrow \mathbb{Y}$ (называемое редукцией) такое, что $x \mathrm{E} y \Longleftrightarrow \vartheta(x) \mathrm{F} \vartheta(y)$ для всех $x, y \in \mathbb{X}$;

(ii) $\mathrm{E} \sim_{\mathrm{B}} \mathrm{F}$ означает, что $\mathrm{E} \leqslant_{\mathrm{B}} \mathrm{F}$ и $\mathrm{F} \leqslant_{\mathrm{B}} \mathrm{E}$ (двусторонняя сводимость);

(iii) $\mathrm{E}<_{\mathrm{B}} \mathrm{F}$ означает, что $\mathrm{E} \leqslant_{\mathrm{B}} \mathrm{F}$, но $\mathrm{F} \underset{\mathrm{B}}{\mathrm{B}} \mathrm{E}$ (строгая сводимость).

Рассматриваются следуюшие операции с отношениями эквивалентности на "польских" пространствах:

(e1) счетное оббединение (если оно дает ОЭ) и счетное пересечение ОЭ на одном и том же пространстве;

(e2) счетная дизбюнктная сумма $\mathrm{F}=\bigvee_{k \in \mathbb{N}} \mathrm{F}_{k}$ данных $\mathrm{O} \mathrm{F}_{k}$ на "польских" пространствах $\mathbb{S}_{k}$ определяется как ОЭ на $\mathbb{S}=\bigcup_{k}\left(\{k\} \times \mathbb{S}_{k}\right)$ (с "польской" топологией, порожденной множествами вида $\{k\} \times U$, где $U \subseteq \mathbb{S}_{k}$ открыто $\left.{ }^{7}\right)$, именно, $\langle k, x\rangle \mathrm{F}\langle l, y\rangle$ выполнено, когда $k=l$ и $x \mathrm{~F}_{k} y$;

(е3) произведение $\mathrm{P}=\prod_{k} \mathrm{~F}_{k}$ данных $\bigcirc$ Э $\mathrm{F}_{k}$ на пространствах $\mathbb{S}_{k}$ есть ОЭ на $\prod_{k} \mathbb{S}_{k}$, определенное так, что $x \mathrm{P} y$ выполнено, когда $x_{k} \mathrm{~F}_{k} y_{k}$ для всех $k$, в частности, если $\mathrm{E}, \mathrm{F}$ суть $\mathrm{O}$ на $\mathbb{X}, \mathbb{Y}$, то $\mathrm{P}=\mathrm{E} \times \mathrm{F}$ определяется на $\mathbb{X} \times \mathbb{Y}$ так, что $\langle x, y\rangle \mathrm{P}\left\langle x^{\prime}, y^{\prime}\right\rangle$, когда $x \mathrm{E} x^{\prime}$ и $y \mathrm{~F} y^{\prime}$;

(е4) произведение Фубини $\mathrm{F}=\prod_{k \in \mathbb{N}} \mathrm{F}_{k} / \mathscr{I}$ данных ОЭ $\mathrm{F}_{k}$ на пространствах $\mathbb{S}_{k}$ по модулю идеала $\mathscr{I}$ на $\mathbb{N}$ есть ОЭ на произведении пространств $\prod_{k \in \mathbb{N}} \mathbb{S}_{k}$, определенное так, что $x \mathrm{~F} y$ выполнено, когда $\left\{k: x_{k} \mathbb{E}_{k} y_{k}\right\} \in \mathscr{I}$;

(е5) счетная степень $\mathrm{F}^{\infty}$ данного ОЭ $\mathrm{F}$ на $\mathbb{S}$ есть ОЭ на $\mathbb{S}^{\mathbb{N}}$, определенное так, что $x \mathrm{~F}^{\infty} y$ выполнено, когда $\left\{\left[x_{k}\right]_{\mathrm{F}}: k \in \mathbb{N}\right\}=\left\{\left[y_{k}\right]_{\mathrm{F}}: k \in \mathbb{N}\right\}$, другими словами,

\footnotetext{
${ }^{7}$ Если $\mathbb{S}_{k}$ попарно дизъюнктны и открыты в $\mathbb{S}^{\prime}=\bigcup_{k} \mathbb{S}_{k}$, то можно равносильным образом определить $\mathrm{F}=\bigvee_{k} \mathrm{~F}_{k}$ на $\mathbb{S}^{\prime}$ так, что $x \mathrm{~F} y$, когда $x, y$ принадлежат одному и тому же $\mathbb{S}_{k}$ и $x \mathrm{~F}_{k} y$.
} 
для всякого $k$ имеется $l$ такое, что выполнено $x_{k} \mathrm{~F} y_{l}$, и для всякого $l$ имеется $k$ такое, что выполнено $x_{k} \mathrm{~F} y_{l}$.

Заметим, что операции (e1), (e2), (e3), (е5) и (е4) с $\mathscr{I}=$ Fin всегда дают борелевское, соответственно, аналитическое ОЭ, когда все данные ОЭ являются борелевскими или аналитическими.

Операции не являются независимыми. Именно, $\bigcap_{k \in \mathbb{N}} \mathrm{F}_{k}$ борелевски сводится к $\prod_{k} \mathrm{~F}_{k}$ отображением $x \mapsto\langle x, x, x, \ldots\rangle$, а дизъюнктная сумма $\bigvee_{k \in \mathbb{N}} \mathrm{F}_{k}$ сводится к $\mathrm{D}(\mathbb{N}) \times \prod_{k} \mathrm{~F}_{k}$ отображением $\langle k, x\rangle \mapsto\left\langle k, x_{0}, \ldots, x_{k-1}, x, x_{k+1}, \ldots\right\rangle$, где $x_{k} \in \mathbb{S}_{k}-$ раз и навсегда фиксированные точки. Само же произведение $\prod_{k \in \mathbb{N}} \mathrm{F}_{k}$ выразимо в терминах произведения Фубини по модулю Fin. В самом деле, рассмотрим функцию $f: \mathbb{N} \stackrel{\text { на }}{\longrightarrow} \mathbb{N}$ такую, что $f^{-1}(n)$ бесконечно для каждого $n$. Положим $\mathrm{E}_{k}=\mathrm{F}_{f(k)}$. Теперь для $x=\left\langle x_{0}, x_{1}, x_{2}, \ldots\right\rangle \in \prod_{k} \mathbb{S}_{k}$ (где $\mathbb{S}_{k}$ - область $\mathrm{F}_{k}$ ) пусть $\vartheta(x)=\left\langle y_{0}, y_{1}, y_{2}, \ldots\right\rangle$, где $y_{k}=x_{f(k)}$. Тогда $\vartheta-$ борелевская редукция $\prod_{k} \mathrm{~F}_{k}$ к $\prod_{k} \mathrm{E}_{k} /$ Fin. Однако произведение Фубини и счетная степень точно не сводятся друг к другу, и мало что известно о счетном объединении в (e1).

Таким образом, класс 4, упомянутый в теоремах 1 и 2, есть наименьший класс, который содержит равенства $\mathrm{D}(\mathbb{S})$ на "польских" пространствах $\mathbb{S}$ и замкнут относительно операций (e1)-(e5) (с $\mathscr{I}=$ Fin в (е4)), а все ОЭ в классе 4 - борелевские на "польских" пространствах.

Сушествует много интересных ОЭ в классе 4, например, семейство ОЭ $\mathrm{T}_{\alpha}$, $\alpha<\omega_{1}$, введенное Х. Фридманом [4], которое начинается с $\mathrm{T}_{0}=\mathrm{D}(\mathbb{N})$ (отношение равенства на $\mathbb{N}$ ), кроме того, $\mathrm{T}_{\alpha+1}=\mathrm{T}_{\alpha}^{\infty}$ для всех $\alpha$ и $\mathrm{T}_{\lambda}=\mathrm{V}_{\alpha<\lambda} \mathrm{T}_{\alpha}$ для предельных ординалов $\lambda$. Таким образом, $\operatorname{dom} \mathrm{T}_{1}=\mathbb{N}^{\mathbb{N}}$ и выполнено $x \mathrm{~T}_{1} y$, когда $\operatorname{ran} x=\operatorname{ran} y$ для $x, y \in \mathbb{N}^{\mathbb{N}}$. Отображение $\vartheta(x)=\chi$, где $\chi$ - характеристическая функция $\operatorname{ran} x$, свидетельствует, что $\mathrm{T}_{1} \leqslant_{\mathrm{B}} \mathrm{D}\left(2^{\mathbb{N}}\right)$. Чтобы доказать обратное неравенство, для $a \in 2^{\mathbb{N}}$ определим $\beta(a)$ как единственную возрастающую биекцию $\mathbb{N} \stackrel{\text { на }}{\rightarrow}|a|=\{k: a(k)=1\}$, если $|a|$ бесконечно, а если $|a|=\left\{k_{0}, \ldots, k_{n}\right\}$, то положим $\beta(a)(i)=k_{i}$ для $i<n$ и $\beta(a)(i)=k_{n}$ для $i \geqslant n$. Функция $\beta$ показывает, что $\mathrm{D}\left(2^{\mathbb{N}}\right) \leqslant_{\mathrm{B}} \mathrm{T}_{1}$, значит, $\mathrm{T}_{1} \sim_{\mathrm{B}} \mathrm{D}\left(2^{\mathbb{N}}\right)$. Отсюда легко следует, что $\mathrm{T}_{2} \sim_{\mathrm{B}} \mathrm{D}\left(2^{\mathbb{N}}\right)^{\infty}$; здесь правая часть часто рассматривается как определение $\mathrm{O} Э \mathrm{~T}_{2}$, которое по этой причине обычно называется равенством счетных множеств вещественных чисел.

1.3. Идеалы. Идеалом на множестве $A$ является любое множество $\varnothing \neq \mathscr{I} \subseteq$ $\mathscr{P}(A)$, замкнутое относительно $\cup$ и удовлетворяющее $x \in \mathscr{I} \wedge y \subseteq x \Longrightarrow y \in \mathscr{I}$. В этом случае $\mathrm{E}_{\mathscr{I}}$ есть $\mathrm{O} Э$ на $\mathscr{P}(A)$, определенное так, что выполнено $X \mathrm{E}_{\mathscr{I}} Y$, когда $X \Delta Y \in \mathscr{I}$. Заметим, что $\mathrm{E}_{\mathscr{I}}$ - борелевское ОЭ, если $\mathscr{I}$ - борелевский идеал. Многие важные $\mathrm{O} Э$ имеют вид $\mathrm{E}_{\mathscr{I}}$, среди них:

$$
\begin{array}{ll}
\mathrm{E}_{0}=\mathrm{E}_{\text {Fin }}, & \text { где } \quad \text { Fin }=\{x \subseteq \mathbb{N}: x \text { конечно }\} ; \\
\mathrm{E}_{1}=\mathrm{E}_{\mathscr{I}_{1}}, & \text { где } \quad \mathscr{I}_{1}=\text { Fin } \times 0=\left\{x \subseteq \mathbb{N}^{2}:\left\{k:(x)_{k} \neq \varnothing\right\} \in \text { Fin }\right\} ; \\
\mathrm{E}_{3}=\mathrm{E}_{\mathscr{I}_{3}}, & \text { где } \quad \mathscr{I}_{3}=0 \times \text { Fin }=\left\{x \subseteq \mathbb{N}^{2}: \forall k\left((x)_{k} \in \text { Fin }\right)\right\} ;
\end{array}
$$

эти три отношения эквивалентности принадлежат классу $4^{8}$. Идеалы вида $\{x \in$

\footnotetext{
${ }^{8}$ Чтобы показать, что $\mathrm{E}_{0}$ принадлежит классу 4 , возьмем при любом $k$ в качестве $\mathrm{F}_{k}$ paвенство на двухэлементном множестве в (е4). Чтобы показать, что Е 3 принадлежит классу 4 , возьмем $\mathrm{P}_{k}=\mathrm{E}_{0}$ в $(\mathrm{e} 3)$.
} 
$\mathscr{P}(\mathbb{N}): x \cap W \in \operatorname{Fin}\}$, где $W \subseteq \mathbb{N}$ бесконечно и кобесконечно, называемые тривиальными вариациями Fin, также порождают ОЭ класса 4.

Пишут $\mathscr{I} \leqslant_{\mathrm{B}} \mathscr{J}, \mathscr{I} \sim_{\mathrm{B}} \mathscr{J}$ и т. д., когда $\mathrm{E}_{\mathscr{I}} \leqslant_{\mathrm{B}} \mathrm{E}_{\mathscr{J}}, \mathrm{E}_{\mathscr{I}} \sim_{\mathrm{B}} \mathrm{E}_{\mathscr{J}}$ и т. д.

Произведение Фубини $\prod_{k \in \mathbb{N}} \mathscr{J}_{k} / \mathscr{I}$ идеалов $\mathscr{J}_{k}$ на множествах $B_{k}$ над идеалом $\mathscr{I}$ на $\mathbb{N}$ - это идеал всех множеств $y \subseteq B=\left\{\langle k, b\rangle: k \in \mathbb{N} \wedge b \in B_{k}\right\}$ таких, что множество $\left\{k:(y)_{k} \notin \mathscr{J}_{k}\right\}$ принадлежит $\mathscr{I}$, где $(y)_{k}=\{b:\langle k, b\rangle \in y\}$ - сечение $y$ (cр. с произведением Фубини отношений эквивалентности). В частности, если $\mathscr{I}$, $\mathscr{J}$ - идеалы на $\mathbb{N}, B$ соответственно, то $\mathscr{I} \otimes \mathscr{J}=\prod_{k \in \mathbb{N}} \mathscr{J}_{k} / \mathscr{I}$, где $\mathscr{J}_{k}=\mathscr{J}$ для всех $k \in \mathbb{N}$, таким образом, $\mathscr{I} \otimes \mathscr{J}$ - идеал всех множеств $y \subseteq \mathbb{N} \times B$ таких, что $\left\{k:(y)_{k} \notin \mathscr{J}\right\} \in \mathscr{I}$.

Идеал $\mathscr{I}$ на $\mathbb{N}$ называется $P$-идеалом, если для любой последовательности множеств $x_{n} \in \mathscr{I}$ существует множество $x \in \mathscr{I}$ такое, что $x_{n} \backslash x \in$ Fin для всех $n$. Например, Fin и $\mathscr{I}_{3}$ (но не $\left.\mathscr{I}_{1}\right)$ - $P$-идеалы.

Этот класс допускает другие характеризации. Субмерой на множестве $A$ называется любая функция $\varphi: \mathscr{P}(A) \rightarrow[0,+\infty]$, удовлетворяюшая $\varphi(\varnothing)=0, \varphi(\{a\})<$ $+\infty$ для всех $a$ и $\varphi(x) \leqslant \varphi(x \cup y) \leqslant \varphi(x)+\varphi(y)$. Субмера $\varphi$ на $\mathbb{N}$ полунепрерывна снизу, или, для краткости, l.s.c. (lower semicontinuous), если $\varphi(x)=$ $\sup _{n} \varphi(x \cap[0, n))$ для всех $x \in \mathscr{P}(\mathbb{N})$. С. Солецки [15] показал, что борелевские $P$-идеалы - это в точности идеалы вида $\operatorname{Exh}_{\varphi}=\left\{x \in \mathscr{P}(\mathbb{N}): \varphi_{\infty}(x)=0\right\}$, где $\varphi-$ l. s. с.-субмера на $\mathbb{N}$ и $\varphi_{\infty}(x)=\inf _{n}(x \cap[n, \infty))$, а также что все борелевские $P$-идеалы - это то же самое, что и "полишабельные" идеалы, т. е. те, которые допускают топологию "польской" групшы с $\Delta$ в качестве операции.

А. Кехрис [11] доказал, что сдвиговые действия борелевских $P$-идеалов $\mathscr{I}$, кроме Fin, $\mathscr{I}_{3}$ и тривиальных вариаций Fin, генерически турбулентны, поэтому соответствуюшие $\mathrm{O} Э \mathrm{E}_{\mathscr{I}}$ принадлежат классу 1.

Семейство Фреше - это наименьшее семейство идеалов $\mathrm{Fr}$, содержащее Fin и замкнутое относительно произведений Фубини $\prod_{n \in \mathbb{N}} \mathscr{I}_{n} /$ Fin. Например, umeрированные идеаль Фреше $\mathscr{J}_{\alpha}$, определенные индукцией по $\alpha<\omega_{1}$ так, что $\mathscr{J}_{0}=$ Fin, $\mathscr{J}_{\alpha+1}=$ Fin $\otimes \mathscr{J}_{\alpha}$ для всех $\alpha$ и $\mathscr{J}_{\lambda}=\prod_{\alpha<\lambda} \mathscr{J}_{\alpha} /$ Fin $_{\lambda}$ для предельных $\lambda$, где $\operatorname{Fin}_{\lambda}$ - идеал всех конечных подмножеств $\lambda$, принадлежат Fr. (Модификация, принятая в [8], использует на каждом предельном шаге $\lambda$ фиксированную конфинальную в $\lambda \omega$-последовательность.)

По определению если $\mathscr{I} \in \mathrm{Fr}$, то $\mathrm{E}_{\mathscr{I}}$ есть $\mathrm{O}$ Һл класса 4.

Через otp $X$ обозначается порядковый тип $X \subseteq$ Ord. Для любых $\alpha, \gamma<\omega_{1}$ множество

$$
\mathscr{I}_{\alpha}^{\gamma}=\left\{A \subseteq \alpha: \operatorname{otp} A<\omega^{\gamma}\right\}
$$

(оно нетривиально только при $\alpha \geqslant \omega^{\gamma}$ ) есть идеал (так как ординалы вида $\omega^{\gamma}$ не являются суммами пары меньших ординалов); эти идеалы, в частности, в случае, когда $\alpha=\omega^{\gamma}$, называются неразложсимыми. Мы не знаем, является ли каждый идеал $\mathscr{I}_{\alpha}^{\gamma}$ действительно изоморфным идеалу из $\mathrm{Fr}$, однако можно доказать, что каждый $\mathscr{I}_{\alpha}^{\gamma}$ борелевски сводится к идеалу из Fr. Аналогично, идеалы Beйсcа

$$
\mathscr{W}_{\alpha}^{\gamma}=\left\{A \subseteq \alpha:|A|_{\mathrm{CB}}<\omega^{\gamma}\right\}
$$

(нетривиальные только при $\alpha \geqslant \omega^{\omega^{\gamma}}$ ), где $|X|_{\mathrm{CB}}$ - ранг Кантора-Бендиксона множества $X \subseteq$ Ord (см. $[2$, п. 1.14]), борелевски сводимы к идеалам из Fr. 


\section{§2. Доказательство теоремы 1}

Доказательство теоремы 1 использует следующие вспомогательные понятия. Пусть Е, F- ОЭ на "польских" пространствах $\mathbb{X}, \mathbb{Y}$ соответственно. Отображение $\vartheta: \mathbb{X} \rightarrow \mathbb{Y}$ называется:

(i) (E, F)-инвариантныц, когда $x$ E $y \Longrightarrow \vartheta(x) \mathrm{F} \vartheta(y)$ для всех $x, y \in \mathbb{X}$;

(ii) генерически ${ }^{9}(\mathrm{E}, \mathrm{F})$-инвариантны.м, если имеем $x \mathrm{E} y \Longrightarrow \vartheta(x) \mathrm{F} \vartheta(y)$ для всех $x, y$ из котошего множества $X \subseteq \mathbb{X}$;

(iii) генерической $\mathrm{F}$-константой, когда $\vartheta(x) \mathrm{F} \vartheta(y)$ выполнено для всех $x, y$ на котощем подмножестве пространства $\mathbb{X}$.

Наконец, OЭ Е называется генерически F-эргодическим (см. [6, п. 3.1]), если каждая измеримая по Бэру $(\mathrm{E}, \mathrm{F})$-инвариантная функция является генерической F-константой.

ПРЕДЛОЖЕнИЕ 4. (i) Если Е генерически F-эргодично и не имеет котощих классов әквивалентности, то $\mathrm{E}$ не сводится $\kappa \mathrm{F}$ при помощи измеримого по Бәру отображения.

(ii) Если Е генерически F-эргодично, то даже каждая измеримая по Бәру генерически (E, F)-инвариантная функиия является генерической F-констанmouั.

Покажем ниже, что любое ОЭ, индуцированное “польским" турбулентньм действием, является генерически F-эргодическим для каждого ОЭ F из класса 4.

2.1. Локальные орбиты и турбулентность. Действием группы $\mathbb{G}$ на $\mathbb{X}$ называется любое отображение $a: \mathbb{G} \times \mathbb{X} \rightarrow \mathbb{X}$, обычно записываемое как $a(g, x)=$ $g \cdot x$, такое, что 1$) e \cdot x=x$ и 2$) g \cdot(h \cdot x)=(g h) \cdot x$. Тогда $\langle\mathbb{X} ; a\rangle$, а также само $\mathbb{X}$ называются $\mathbb{G}$-пространством. Непрерывное действие "польской" группы $10 \mathbb{G}$ на "польском" пространстве $\mathbb{X}$ назьвается "польским" действием, а само $\mathbb{X}$

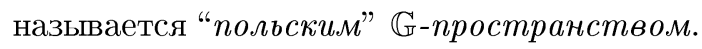

Любое действие $a$ группы $\mathbb{G}$ на $\mathbb{X}$ индуцирует “орбитальное” ОЭ $\mathrm{E}_{a}^{\mathbb{X}}=\mathrm{E}_{\mathbb{G}}^{\mathbb{X}}$, определенное на $\mathbb{X}$ так, что выполнено $x \mathbb{E}_{\mathbb{G}}^{\mathbb{X}} y$, когда имеется $g \in \mathbb{G}$, удовлетворяющее равенству $y=g \cdot x$; его классы эквивалентности

$$
[x]_{\mathbb{G}}=[x]_{\mathbb{E}_{\mathbb{G}}^{\mathbb{X}}}=\{y: \exists g \in \mathbb{G}(g \cdot x=y)\}
$$

- $\mathbb{G}$-орбиты. Индуцированные ОЭ “польских" действий-аналитические (как множества пар), иногда даже борелевские $[1, \S 7]$.

Допустим, что группа $\mathbb{G}$ действует на пространстве $\mathbb{X}$. Если $G \subseteq \mathbb{G}$ и $X \subseteq \mathbb{X}$, то положим

$$
\mathrm{R}_{G}^{X}=\left\{\langle x, y\rangle \in X^{2}: \exists g \in G(x=g \cdot y)\right\},
$$

и пусть $\sim_{G}^{X}$ обозначает ОЭ-оболочку $\mathrm{R}_{G}^{X}$, т. е. $\subseteq$-наименьшее $Э Э$ на $X$ такое, что $x \mathrm{R}_{G}^{X} y \Longrightarrow x \sim_{G}^{X} y$. В частности, $\sim_{\mathbb{G}}^{\mathbb{X}}=\mathbb{E}_{\mathbb{G}}^{\mathbb{X}}$, но, вообше говоря, $\sim_{G}^{X} \varsubsetneqq \mathrm{E}_{\mathbb{G}}^{\mathbb{X}} \mid X$.

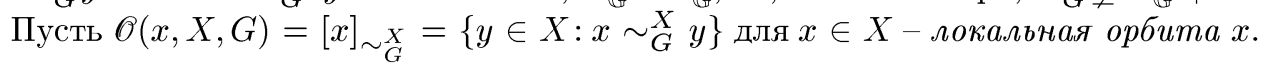
В частности, $[x]_{\mathbb{G}}=[x]_{\mathbb{E}_{\mathbb{G}}^{\mathbb{X}}}=\mathscr{O}(x, \mathbb{X}, \mathbb{G})$ - полная $\mathbb{G}$-орбита $x \in \mathbb{X}$.

\footnotetext{
${ }^{9}$ В работах в этой области, термин "генерически" означает то, что свойство выполнено на котощей области.

${ }^{10}$ Т. е. топологической группы, чье базовое множество есть "польское" пространство, а групповая операция и отображение, дающее обратный элемент, непрерывны.
} 
ОпРЕДЕЛЕнИЕ 5 (вариант Кехриса $[13, \S 8]$ ). Допустим, что $\mathbb{X}$ - "польское" пространство и $\mathbb{G}$ - "польская" группа, непрерьвно действующая на $\mathbb{X}$. Тогда:

1) точка $x \in \mathbb{X}$ турбулентна, когда для любого открытого непустого множества $X \subseteq \mathbb{X}$, содержащего $x$, и любой окрестности $G \subseteq \mathbb{G}$ элемента $1_{\mathbb{G}}(G$ - необязательно подгруппа) локальная орбита $\mathscr{O}(x, X, G)$ где-то плотна (т. е. не является нигде не плотным множеством) в $\mathbb{X}$;

2 ) орбита $[x]_{\mathbb{G}}$ турбулентна, если точка $x$ турбулентна (тогда все точки $y \in$ $[x]_{\mathbb{G}}$ турбулентны);

3) действие $(\mathbb{G}$ на $\mathbb{X})$ турбулентно, а $\mathbb{X}$ - турбулентное "польское" $\mathbb{G}$-пространство, если все орбиты плотны и тощие; действие генерически турбулентно, а $\mathbb{X}$ - генерически турбулентное "польское" $\mathbb{G}$-пространство, если объединение всех плотных турбулентных тощих орбит $[x]_{\mathbb{G}}$ является котощим множеством.

ОЭ, индуцированные генерически турбулентными “польскими” действиями, составляют класс 1 из теоремы 1. Чтобы доказать теорему 1, покажем, что все ОЭ в классе 1 генерически F-эргодичны для каждого F класса 4. Метод доказательства использует индукцию по построению ОЭ класса 4. Некоторым неудобством является то, что мы должны рассмотреть чуть более сильное свойство в рамках индуктивной схемы.

Допустим, что $\mathrm{F}$ - ОЭ на "польском" пространстве. Действие $\mathbb{G}$ на $\mathbb{X}$ называется наследственно генерически $\mathrm{F}$-эргодическим, если $\mathrm{O} \sim_{G}^{X}$ генерически F-эргодично всякий раз, когда $X \subseteq \mathbb{X}$ - непустое открытое множество, $G \subseteq \mathbb{G}$ - непустое открытое множество, содержащее $1_{\mathfrak{G}}$, и локальные орбиты $\mathscr{O}(x, X, G)$ плотны в $X$ для котошего (в $X)$ множества точек $x \in X$. Это, очевидно, влечет генерическую F-эргодичность, если действие генерически турбулентно.

ТеОрема 6. Пусть $\mathbb{G}$ - "польская" группа, а $\mathbb{X}$ - генерически турбулентное "польское" $\mathbb{G}$-пространство. Тогда отночение $\mathbb{E}_{\mathbb{G}}^{\mathbb{X}}$ наследственно генерически F-әргодично, значит, согласно предложсению 4 оно не сводимо ни к какому ОЭ F класса 4 измеримым по Бәру отображсением.

2.2. Предварительный материал к доказательству теоремы 6. Сформулируем два достаточно простых технических факта, относящихся к турбулентности.

ЛЕмма 7. В условиях теоремы 6 пусть непустое множество $X \subseteq \mathbb{X}$ открыто, $G \subseteq \mathbb{G}$ - окрестность $1_{\mathbb{G}}$ и $\mathscr{O}(x, X, G)$ плотно в $X$ для $X$-котощего множества точек $x \in X$. Тогда если $U, U^{\prime} \subseteq X$ открыты и непусты, а $D \subseteq X$ котощее в $X$, то существуют точки $x \in D \cap U$ u $x^{\prime} \in D \cap U^{\prime}$ такие, что $x \sim \sim_{G}^{X} x^{\prime}$.

ДоКАЗАТЕЛЬСТво. В наших предположениях найдутся точки $x_{0} \in U$ и $x_{0}^{\prime} \in$ $U^{\prime}$, удовлетворяюшие соотношению $x_{0} \sim_{G}^{X} x_{0}^{\prime}$, т. е. $x_{0}^{\prime}=g_{n} g_{n-1} \ldots g_{1} \cdot x_{0}$ для набора элементов $g_{1}, \ldots, g_{n} \in G$ таких, что $g_{k} \ldots g_{1} \cdot x_{0} \in X$ для всех $k \leqslant n$. Поскольку рассматриваемое действие непрерьвно, найдется окрестность $U_{0} \subseteq U$ точки $x_{0}$ такая, что $g_{k} \ldots g_{1} \cdot x \in X$ и $g_{n} g_{n-1} \ldots g_{1} \cdot x \in U_{2}$ для всех $x \in U_{0}$. Поскольку $D$ котощее, ясно, что сушествует точка $x \in U_{0} \cap D$, удовлетворяюшая $x^{\prime}=g_{n} g_{n-1} \ldots g_{1} \cdot x \in U^{\prime} \cap D$. Лемма доказана. 
ЛЕмма 8. В условиях теоремы 6 для любых открытых непустых множеств $U \subseteq \mathbb{X} u G \subseteq \mathbb{G}, 1_{\mathbb{G}} \in G$, имеется открытое непустое множество $U^{\prime} \subseteq U$ такое, что локальные орбитьи $\mathscr{O}\left(x, U^{\prime}, G\right)$ плотнь в $U^{\prime}$ для $U^{\prime}$-котощего множества $x \in U^{\prime}$.

ДокаЗАТЕЛьство. Через Int $\bar{X}$ обозначается внутренность замыкания $X$. Если $x \in U$ и множество $\mathscr{O}(x, U, G)$ где-то плотно (в $U)$, то множество $U_{x}=U \cap$ Int $\overline{\mathscr{O}(x, U, G)} \subseteq U$ открыто и $\sim{ }_{G}^{U}$-инвариантно (наблюдение, сделанное, например, в $\left[13\right.$, доказательство 8.4]), кроме того, $\mathscr{O}(x, U, G) \subseteq U_{x}$, так что $\mathscr{O}(x, U, G)=$ $\mathscr{O}\left(x, U_{x}, G\right)$. Из инвариантности орбит следует, что множества $U_{x}$ попарно дизъюнктны, а вследствие турбулентности их объединение плотно в $U$. Рассмотрим любое непустое $U_{x}$ в качестве искомого $U^{\prime}$. Лемма доказана.

Доказательство теоремы 6 проводится индукцией по определению ОЭ в классе 4 операциями, упомянутыми в п. 1.2, в нескольких последуюших пунктах. Начнем с базы индукции: докажем, что в предположениях теоремы $\mathbb{E}_{\mathbb{G}}^{\mathbb{X}}$ является наследственно генерически $\mathrm{D}(\mathbb{N})$-эргодическим. Пусть $X \subseteq \mathbb{X}$ и $G \subseteq \mathbb{G}$ - непустые открытые множества, $1_{\mathbb{G}} \in G$, и локальные орбиты $\mathscr{O}(x, X, G)$ плотны в $X$ для $X$-котошего множества точек $x \in X$. Докажем, что отношение $\sim_{G}^{X}$ генерически $\mathrm{D}(\mathbb{N})$-эргодично.

Рассмотрим измеримое по Бэру генерически $(\sim \underset{G}{X}, \mathrm{D}(\mathbb{N}))$-инвариантное отображение $\vartheta: \mathbb{X} \rightarrow \mathbb{N}$. Пусть, напротив, $\vartheta$ не является генерической $\mathrm{D}(\mathbb{N})$-константой. Тогда существуют открытые непустые множества $U_{1}, U_{2} \subseteq X$, числа $\ell_{1} \neq \ell_{2}$ и котощее множество $D \subseteq X$ такие, что $\vartheta(x)=\ell_{1}$ для всех $x \in D \cap U_{1}$ и $\vartheta(x)=\ell_{2}$ для всех $x \in D \cap U_{2}$. Согласно лемме 7 найдется пара точек $x_{1} \in U_{1} \cap D$ и $x_{2} \in U_{2} \cap D$, удовлетворяюших соотношению $x_{1} \sim_{G}^{X} x_{2}$. Противоречие.

2.3. Индуктивный шаг счетной степени. Рассмотрим генерически турбулентное “польское" $\mathbb{G}$-пространство $\mathbb{X}$ и борелевское ОЭ F на "польском" пространстве $\mathbb{Y}$. Предполагая, что действие $\mathbb{G}$ на $\mathbb{X}$ наследственно генерически F-эргодично, докажем, что это действие наследственно генерически $\mathrm{F}^{\infty}$-эргодично. Фиксируем непустое открытое множество $X_{0} \subseteq \mathbb{X}$ и окрестность $G_{0}$ элемента $1_{\mathbb{G}}$ в $\mathbb{G}$ такие, что $\mathscr{O}\left(x, X_{0}, G_{0}\right)$ плотно в $X_{0}$ для $X_{0}$-котошего множества точек $x \in X_{0}$. Покажем, что любая заданная измеримая по Бэру $\left(\sim_{G_{0}}^{X_{0}}, \mathrm{~F}^{\infty}\right)$-инвариантная функция $\vartheta: X_{0} \rightarrow \mathbb{Y}^{\mathbb{N}}$ - генерическая $\mathrm{F}^{\infty}$-константа. По определению имеем

$$
\text { для } x, x^{\prime} \in X_{0}: \quad x \sim_{G_{0}}^{X_{0}} x^{\prime} \Longrightarrow \forall k \exists l\left(\vartheta_{k}(x) \mathrm{F} \vartheta_{l}\left(x^{\prime}\right)\right) \text {, }
$$

где $\vartheta_{k}(x)=\vartheta(x)(k)$.

Заметим, что $\vartheta$ непрерьвна на плотном $\mathbf{G}_{\delta}$-множестве $D \subseteq X_{0}$.

ЛЕмма 9. Для всякого числа $k$ и любого открытого непустого множества $U \subseteq X_{0}$ существует открытое непустое множество $W \subseteq U$ такое, что $\vartheta_{k}$ есть генерическая $\mathrm{F}-$ константа на $W$.

ДокАЗАТЕЛЬСтво. Используя рассуждения, связанные с категорией и соотношением (1), получаем число $l$, открытые непустые множества $W \subseteq U, Q \subseteq G_{0}$ и плотное в $W \times Q$ множество $P \subseteq W \times Q$ класса $\mathbf{G}_{\delta}$ такое, что $\vartheta_{k}(x) \mathrm{F} \vartheta_{l}(g \cdot x)$ выполнено для всех пар $\langle x, g\rangle \in P$. Можно считать, что $\langle x, g\rangle \in P \Longrightarrow x \in D$. 
Поскольку $Q$ открыто, сушествуют элемент $g_{0} \in Q$ и окрестность $G \subseteq G_{0}$ элемента $1_{\mathbb{G}}, G^{-1}=G$, такие, что $g_{0} G \subseteq Q$.

Продолжение доказательства использует метод форсинга ${ }^{11}$.

Фиксируем счетную транзитивную модель $\mathfrak{M}$ теории ZFHC, т. е. ZFC без аксиомы степени, но с аксиомой, постулируюшей, что каждое множество наследственно счетно. Можно предполагать, что $\mathbb{X}$ имеет код в $\mathfrak{M}$ в том смысле, что существует множество $D_{\mathbb{X}} \in \mathfrak{M}$, являюшееся плотным (счетным) подмножеством $\mathbb{X}$, и $d_{\mathbb{X}} \nmid D_{\mathbb{X}}$ (функция расстояния $\mathbb{X}$, ограниченная на $D_{\mathbb{X}}$ ) также принадлежит $\mathfrak{M}$. Предполагается, что $\mathbb{G}, \mathbb{Y}$, действие $\mathbb{G}$ на $\mathbb{X}$, множества $G, D_{\mathbb{X}}, P$ и отображение $\vartheta \mid D_{\mathbb{X}}$ имеют коды в $\mathfrak{M}$ в аналогичном смысле.

Рассмотрим форсинг Коэна $\mathbf{C}_{\mathbb{X}}$ для $\mathbb{X}$, состоящий из рациональных шаров с центрами в фиксированном плотном счетном подмножестве $\mathbb{X}$, и форсинг Коэна $\mathbf{C}_{\mathbb{G}}$ для $\mathbb{G}$, определенный аналогично. Как обычно, $U \subseteq V$ означает, что $U$ - более сильное "вынуждаюшее условие". В наших допушениях понятие генерической по Коэну над $\mathfrak{M}$ точки $\mathbb{X}$ или $\mathbb{G}$ имеет смысл, а множество всех генерических по Коэну над $\mathfrak{M}$ точек $\mathbb{X}$ есть плотное $\mathbf{G}_{\delta}$-подмножество $\mathbb{X}$, включенное в $D$.

УТВЕРЖДЕНИЕ 10 (ключевой момент турбулентности). Если точки $x, x^{\prime} \in W$ являются $\mathbf{C}_{\mathbb{X}}$-генерическими над $\mathfrak{M}$ и $x \sim_{G}^{W} x^{\prime}$, то $\vartheta_{k}(x) \mathrm{F} \vartheta_{k}\left(x^{\prime}\right)$.

ДокАЗАТЕЛЬСТво. Проведем доказательство индукцией по числу $n\left(x, x^{\prime}\right)$, равному наименьшему $n$ такому, что имеются элементы $g_{1}, \ldots, g_{n} \in G$, удовлетворяюшие соотношению

$$
x^{\prime}=g_{n} g_{n-1} \ldots g_{1} \cdot x \text { и } g_{k} \ldots g_{1} \cdot x \in W \quad \text { для всех } k \leqslant n .
$$

Пусть $n\left(x, x^{\prime}\right)=1$, т.е. $x=h \cdot x^{\prime}$ для некоторого $h \in G \cap \mathfrak{M}\left[x, x^{\prime}\right]{ }^{12}$. Рассмотрим любой $\mathbf{C}_{\mathbb{G}}$-генерический над $\mathfrak{M}\left[x, x^{\prime}\right]$ элемент $g \in Q$, достаточно близкий к $g_{0}$ для того, чтобы $g^{\prime}=g h^{-1}$ принадлежало $Q$. Пара $\langle x, g\rangle$ является $\left(\mathbf{C}_{\mathbb{X}} \times \mathbf{C}_{\mathbb{G}}\right)$-генерической над $\mathfrak{M}$ по теореме о произведении форсингов, откуда $\langle x, g\rangle \in P$ (ибо $P$ - плотное $\mathbf{G}_{\delta}$-множество с кодом в $\left.\mathfrak{M}\right)$ и $\vartheta_{k}(x) \mathrm{F} \vartheta_{l}(g \cdot x)$ по выбору $P$. Более того, элемент $g^{\prime}$ сам $\mathbf{C}_{\mathbb{G}}$-генеричен над $\mathfrak{M}\left[x^{\prime}\right]$, откуда $\vartheta_{k}\left(x^{\prime}\right) \mathrm{F} \vartheta_{l}\left(g^{\prime} \cdot x^{\prime}\right)$ выводится по тем же соображениям. Однако $g^{\prime} \cdot x^{\prime}=g h^{-1}(h \cdot x)=g \cdot x$.

Теперь допустим, что (2) выполнено для некоторого $n \geqslant 2$. Возьмем $\mathbf{C}_{\mathbb{G}}$-генерический над $\mathfrak{M}\left[x, x^{\prime}\right]$ элемент $g_{1}^{\prime} \in G$, достаточно близкий к $g_{1}$, чтобы $g_{2}^{\prime}=g_{2} g_{1} g_{1}^{\prime-1}$ принадлежало $G$, а точка $x^{*}=g_{1}^{\prime} \cdot x$ принадлежала $W$. Тогда точка $x^{*} \mathbf{C}_{\mathbb{X}}$-генерична над $\mathfrak{M}$ (произведение форсингов) и $n\left(x^{*}, x^{\prime}\right) \leqslant n-1$, ибо $g_{2}^{\prime} \cdot x^{*}=g_{2} g_{1} \cdot x$. Утверждение доказано.

Итак, доказано, что функция $\vartheta_{k}$ является генерически $\left(\sim_{G}^{W}, \mathrm{~F}\right)$-инвариантной на $W$ (т. е. инвариантной на котощем подмножестве $W$ ). Можно также предположить в силу леммы 8 , что орбита $\mathscr{O}(x, W, G)$ плотна в $W$ для $W$-котощего множества точек $x \in W$. Тогда в силу наследственной генерической $\mathrm{F}$-эргодичности

\footnotetext{
${ }^{11}$ Предполагается некоторое знакомство читателя с методом форсинга. Лемма может быть доказана и чисто топологически, но при этом рассуждения теряют прозрачность.

12 Десь $\mathfrak{M}\left[x, x^{\prime}\right]$ определяется не как генерическое расширение $\mathfrak{M}$, а как любая (счетная транзитивная) модель ZFHC, содержащая $x, x^{\prime}$ и все множества из $\mathfrak{M}$. Модель $\mathfrak{M}\left[x, x^{\prime}\right]$ может содержать больше ординалов, чем $\mathfrak{M}$, но это для нас не так важно.
} 
$\vartheta_{k}$ является генерической F-константой на $W$, что и требовалось доказать. Лемма 9 доказана.

Согласно доказанной лемме 9 существуют котощее в $X_{0}$ множество $Z \subseteq X_{0}$ и счетное множество $Y=\left\{y_{j}: j \in \mathbb{N}\right\} \subseteq \mathbb{Y}$ такие, что для любого $k$ и любого $x \in Z$ имеется $j$ такое, что $\vartheta_{k}(x) \mathrm{F} y_{j}$. Положим $\eta(x)=\bigcup_{k \in \mathbb{N}}\left\{j: \vartheta_{k}(x) \mathrm{F} y_{j}\right\}$. Тогда для любой пары $x, x^{\prime} \in Z$ соотношение $\vartheta(x) \mathrm{F}^{\infty} \vartheta\left(x^{\prime}\right)$ равносильно $\eta(x)=\eta\left(x^{\prime}\right)$, так что согласно инвариантности $\vartheta$ имеем

$$
x \sim_{G_{0}}^{X_{0}} x^{\prime} \Longrightarrow \eta(x)=\eta\left(x^{\prime}\right) \quad \text { для всех } x, x^{\prime} \in Z .
$$

Остается доказать, что $\eta$ - константа на котощем подмножестве $Z$.

Предположим, что, напротив, сушествуют два непустых открытых множества $U_{1}, U_{2} \subseteq X_{0}$, число $j \in \mathbb{N}$ и котощее множество $Z^{\prime} \subseteq Z$ такие, что $j \in \eta\left(x_{1}\right)$ и $j \notin \eta\left(x_{2}\right)$ для всех $x_{1} \in Z^{\prime} \cap U_{1}$ и $x_{2} \in Z^{\prime} \cap U_{2}$. Теперь лемма 7 противоречит соотношению (3), что доказывается так же, как в конще п. 2.2 .

2.4. Индуктивный шаг произведения Фубини. Пусть $\mathbb{X}$ - генерически турбулентное "польское" $\mathbb{G}$-пространство. Докажем, что действие $\mathbb{G}$ на $\mathbb{X}$ наследственно генерически $\mathrm{F}$-эргодично, где $\mathrm{F}=\prod_{k} \mathrm{~F}_{k} / \mathrm{Fin}, \mathrm{F}_{k}$ - борелевские ОЭ на "польских" пространствах $\mathbb{Y}_{k}$ и действие наследственно генерически $\mathrm{F}_{k}$-эргодично для каждого $k$. Фиксируем открытое непустое множество $X_{0} \subseteq \mathbb{X}$ и окрестность $G_{0}$ элемента $1_{\mathbb{G}}$ в $\mathbb{G}$ такие, что $\mathscr{O}\left(x, X_{0}, G_{0}\right)$ плотно в $X_{0}$ для $X_{0}$-котошего множества точек $x \in X_{0}$. Докажем, что любая $\left(\sim_{G_{0}}^{X_{0}}, \mathrm{~F}\right)$-инвариантная измеримая по Бэру функция $\vartheta: X_{0} \rightarrow \mathbb{Y}$ есть генерическая F-константа на $X_{0}$. По определению

$$
\text { для } x, y \in X_{0}: \quad x \sim_{G_{0}}^{X_{0}} y \Longrightarrow \exists k_{0} \forall k \geqslant k_{0}\left(\vartheta_{k}(x) \mathrm{F}_{k} \vartheta_{k}(y)\right),
$$

где $\vartheta_{k}(x)=\vartheta(x)(k)$.

Заметим, что $\vartheta$ непрерьвна на плотном $\mathbf{G}_{\delta}$-множестве $D \subseteq X_{0}$.

ЛЕмма 11. Для всякого открытого непустого множества $U \subseteq X_{0}$ найдутся число $k_{0}$ и открытое непустое множество $W \subseteq U$ такие, что $\vartheta_{k}$ есть генерическая F-константа на $W$ для всех $k \geqslant k_{0}$.

ДоКАЗАТЕЛЬСТво. Используя соотношение (4), легко находим число $k_{0}$, открытые непустые множества $W \subseteq U, Q \subseteq G_{0}$ и плотное в $W \times Q$ множество $P \subseteq W \times Q$ класса $\mathbf{G}_{\delta}$ такие, что $\vartheta_{k}(x) \mathrm{F} \vartheta_{k}(g \cdot x)$ выполнено для всех $k \geqslant k_{0}$ и всех пар $\langle x, g\rangle \in P$. Можно считать, что $\langle x, g\rangle \in P \Longrightarrow x \in D$. Поскольку $Q$ открыто, сушествуют элемент $g_{0} \in Q$ и окрестность $G \subseteq G_{0}$ элемента $1_{\mathbb{G}}, G^{-1}=G$, такие, что $g_{0} G \subseteq Q$.

Пусть модель $\mathfrak{M}$ такова, как в доказательстве леммы 9. Аналогично утверждению 10 докажем, что если точки $x, x^{\prime} \in W$ являются $\mathbf{C}_{\mathbb{X}}$-генерическими над $\mathfrak{M}$, $k \geqslant k_{0}$ и $x \sim_{G}^{W} x^{\prime}$, то $\vartheta_{k}(x) \mathrm{F}_{k} \vartheta_{k}\left(x^{\prime}\right)$, иньпи словами, каждая функция $\vartheta_{k}, k \geqslant k_{0}$, генерически $\left(\sim_{G}^{W}, \mathrm{~F}_{k}\right)$-инвариантна на $W$. Можно предполагать согласно лемме 8 , что некоторое $W$-котощее множество орбит $\mathscr{O}(x, W, G)$ плотно в $W$. Теперь в силу наследственно генерической $\mathrm{F}_{k}$-эргодичности все отображения $\vartheta_{k}, k \geqslant k_{0}$, являются генерическими $\mathrm{F}_{k}$-константами на $W$, что и требовалось доказать. Лемма доказана. 
Понятно, что если $W$ выбрано так же, как в лемме 11 , то $\vartheta$ есть генерическая F-константа на $W$. Остается показать, что эти константы F-эквивалентны друг другу. Пусть, напротив, сушествуют два непустых открытых множества $W_{1}, W_{2} \subseteq X_{0}$ и пара точек $y \forall y^{\prime}$ в $\mathbb{Y}$ такие, что $\vartheta(x) \mathrm{F} y$ и $\vartheta\left(x^{\prime}\right) \mathrm{F} y^{\prime}$ для котоших множеств $x \in W_{1}$ и $x^{\prime} \in W_{2}$. Противоречие получается тем же методом, что и в конце п. 2.3.

2.5. Остальные индуктивные шаги. Теперь рассматриваются операции (e1), (e2), (е3) из п. 1.2.

Для рассмотрения счетного объединения предположим, что $\mathrm{F}_{1}, \mathrm{~F}_{2}, \mathrm{~F}_{3}, \ldots$ - борелевские ОЭ на "польском" пространстве $\mathbb{Y}, \mathrm{F}=\bigcup_{k} \mathrm{~F}_{k}$ является ОЭ, а "польское" и генерически турбулентное действие $\mathbb{G}$ на $\mathbb{X}$ наследственно генерически $\mathrm{F}_{k}$-эргодично для каждого $k$, и докажем, что действие остается наследственно генерически F-эргодическим.

Фиксируем непустое открытое множество $X_{0} \subseteq \mathbb{X}$ и окрестность $G_{0}$ элемента $1_{\mathbb{G}}$ в $\mathbb{G}$ такие, что $X_{0}$-котощее множество орбит $\mathscr{O}\left(x, X_{0}, G_{0}\right), x \in X_{0}$, плотны в $U_{0}$. Рассмотрим $\left(\sim_{G_{0}}^{X_{0}}, \mathrm{~F}\right)$-инвариантную измеримую по Бэру функцию $\vartheta: X_{0} \rightarrow \mathbb{Y}$, непрерывную на плотном $\mathbf{G}_{\delta}$-множестве $D \subseteq X_{0}$. Из инвариантности $\vartheta$ следует, что для всякого открытого непустого множества $U \subseteq X_{0}$ найдутся число $k$ и открытые непустые множества $W \subseteq U, Q \subseteq G_{0}$ такие, что $\vartheta(x) \mathrm{F}_{k} \vartheta(g \cdot x)$ выполнено для любой $\left(\mathbf{C}_{\mathbb{X}} \times \mathbf{C}_{\mathbb{G}}\right)$-генерической над $\mathfrak{M}$ пары $\langle x, g\rangle \in W \times Q$. Можно найти элемент $g_{0} \in Q \cap \mathfrak{M}$ и окрестность $G \subseteq G_{0}$ элемента $1_{\mathbb{G}}$ такие, что $g_{0} G \subseteq Q$. Как и в доказательстве утверждения 10 , мы имеем $\vartheta(x) \mathrm{F}_{k} \vartheta\left(x^{\prime}\right)$ для всех $\mathbf{C}_{\mathbb{X}}$-генерических над $\mathfrak{M}$ точек $x, x^{\prime} \in W$, удовлетворяющих $x \sim_{G}^{W} x^{\prime}$. Следовательно, в силу эргодичности, $\vartheta$ есть генерическая $\mathrm{F}_{k}$-константа, значит, и генерическая $\mathrm{F}$-константа на $W$. То, что эти $\mathrm{F}$-константы $\mathrm{F}$-эквивалентны друг другу, демонстрируется тем же методом, как в конце п. 2.3. Операция счетного пересечения рассматривается аналогично.

Мы видели в п. 1.2, что операция счетного произведения сводится к произведению Фубини, однако имеется и простое независимое рассуждение. Если $\mathrm{F}_{k}-\mathrm{O}$ на пространствах $\mathbb{Y}_{k}$, то $\mathrm{F}=\prod_{k} \mathrm{~F}_{k}$ - ОЭ на пространстве $\mathbb{Y}=\prod_{k} \mathbb{Y}_{k}$. Отображение $\vartheta: \mathbb{X} \rightarrow \mathbb{Y}(\mathrm{E}, \mathrm{F})$-инвариантно (где $\mathrm{E}$ - любое ОЭ на $\mathbb{X})$, если и только если каждое координатное отображение $\vartheta_{k}(x)=\vartheta(x)(k)\left(\mathrm{E}, \mathrm{F}_{k}\right)$-инвариантно, из чего немедленно следует искомый результат.

Операция дизъюнктной суммы сводится к произведению (см. п. 1.2).

Таким образом, теорема 6 и теорема 1 доказаны.

\section{§ 3. Приложения}

Этот параграф̆ содержит два приложения теоремы 6. Одно из них - это теорема 2. Другое показывает, как теорема Хъёрта, упомянутая во введении (о том, что “турбулентные” ОЭ борелевски несводимы к "польским" действиям группы $S_{\infty}$ всех перестановок $\mathbb{N}$ ), может быть получена из теоремы 6 достаточно простыми рассуждениями.

3.1. Доказательство теоремы 2. Фиксируем нетривиальный, как в теореме 2, борелевский $P$-идеал $\mathscr{Z} \subseteq \mathscr{P}(\mathbb{N})$. Согласно теореме Солецки (см. п.1.3) су- 
шествует 1. s. с-субмера $\varphi$ на $\mathbb{N}$ такая, что $\mathscr{Z}=\left\{x \subseteq \mathbb{N}: \varphi_{\infty}(x)=0\right\}$. Положим $r_{k}=\varphi(\{k\})$.

Лемма 12 [11]. Если $\mathscr{Z}$ отлично от Fin, не является тривиальной вариацией Fin и не изоморфно $\mathscr{I}_{3}=0 \times$ Fin, то найдется множество $W \notin \mathscr{Z}$ maкое, что $\left\{r_{k}\right\}_{k \in W} \rightarrow 0$.

ДокАЗАТЕЛЬСТво. Пусть $U_{n}=\left\{k: r_{k} \leqslant \frac{1}{n}\right\}$ и $U_{0}=\mathbb{N}$, таким образом, $U_{n+1} \subseteq U_{n}$ для всех $n$. Мы утверждаем, что $\inf _{m \in \mathbb{N}} \varphi\left(U_{m}\right)>0$. Иначе любое множество $x \subseteq \mathbb{N}$ принадлежит $\mathscr{Z}$, если и только если $x \backslash U_{n}$ конечно для каждого $n$. Если множество $N=\left\{n: U_{n} \backslash U_{n+1}\right.$ бесконечно $\}$ пусто, то, очевидно, $\mathscr{Z}=\mathscr{P}(\mathbb{N})$. Если $N \neq \varnothing$ конечно, то $\mathscr{Z}$ есть либо Fin (если $U_{n}=\varnothing$ для почти всех $n$ ), либо тривиальная вариация Fin (если $U_{n}$ непусто для всех $n$ ). Наконец, если $N$ бесконечно, то $\mathscr{Z}$ изоморфно $0 \times$ Fin (например, если все множества $D_{n}=U_{n} \backslash U_{n+1}$ бесконечны, то $x \in \mathscr{Z}$, когда $x \cap D_{n}$ конечно для всех $\left.n\right)$. Итак, мы имеем во всех случаях противоречие с предположениями леммы.

Значит, существует $\varepsilon>0$ такое, что $\varphi\left(U_{m}\right)>\varepsilon$ для всех $m$. Поскольку $\varphi$ является 1.s. c.-субмерой, можно определить возрастающую последовательность чисел $n_{1}<n_{2}<n_{3}<\cdots$ и для каждого $l$ конечное множество $w_{l} \subseteq U_{n_{l}} \backslash U_{n_{l+1}}$ такое, что $\varphi\left(w_{l}\right)>\varepsilon$. Тогда $W=\bigcup_{l} w_{l} \notin \mathscr{Z}$ и, очевидно, $\left\{r_{k}\right\}_{k \in W} \rightarrow 0$. Лемма доказана.

Поскольку $\mathrm{E}_{\mathscr{Z} \mid W} \leqslant{ }_{\mathrm{B}} \mathrm{E}_{\mathscr{Z}}$, то теперь для теоремы 2 достаточно доказать следующую лемму.

Лемма 13. Если $\mathscr{Z}, \varphi, r_{k}$ - такие же, как и выле, $u\left\{r_{k}\right\} \rightarrow 0$, mo сдвиговое действие $\mathscr{Z}$ на $\mathscr{P}(\mathbb{N})$ генерически турбулентно.

ДокАЗАТЕЛЬСТво. $\mathscr{Z}$ - "польская" группа (с операцией $\Delta)$ в топологии $\tau$, индуцированной метрикой $r(x, y)=\varphi(x \Delta y)$. Действие $\mathscr{Z}$ операцией $\Delta$ на пространстве $\mathscr{P}(\mathbb{N})$ (рассматриваемом с топологией произведения через идентификацию с $2^{\mathbb{N}}$ ) непрерьвно. Остается проверить турбулентность.

Пусть $x \in \mathscr{P}(\mathbb{N})$. Легко видеть, что орбита $[x]_{\mathscr{Z}}=\mathscr{Z} \Delta x$ плотна и является множеством первой категории, так что остается проверить, что $x$ - турбулентная точка. Рассмотрим открытое множество $X \subseteq \mathscr{P}(\mathbb{N})$, содержащее $x$, и $\tau$-окрестность $G$ нейтрального элемента $\varnothing$ группь $\mathscr{Z}$; можно предполагать, что для некоторого $k$ выполнено $X=\{y \in \mathscr{P}(\mathbb{N}): y \cap[0, k)=u\}$, где $u=x \cap[0, k)$, и $G=\{g \in \mathscr{Z}: \varphi(g)<\varepsilon\}$ для некоторого $\varepsilon>0$. Докажем, что локальная орбита $\mathscr{O}(x, X, G)$ где-то плотна (т. е. не является нигде не плотной) в $X$.

Возьмем число $l \geqslant k$, достаточно большое для $r_{n}<\varepsilon$ при всех $n \geqslant l$. Пусть $v=x \cap[0, l)$; покажем, что орбита $\mathscr{O}(x, X, G)$ плотна во множестве $Y=\{y \in$ $\mathscr{P}(\mathbb{N}): y \cap[0, l)=v\}$. Рассмотрим открытое множество $Z=\{z \in Y: z \cap[l, j)=w\}$, где $j \geqslant l, w \subseteq[l, j)$. Через $z$ обозначим единственный элемент $Z, z \cap[j,+\infty)=x \cap$ $[j,+\infty)$; тогда $x \Delta z=\left\{l_{1}, \ldots, l_{m}\right\} \subseteq[l, j)$. Каждое $g_{i}=\left\{l_{i}\right\}$ принадлежит $G$ по выбору $l$ (в самом деле, $l_{i} \geqslant l$ ). Более того, элемент $x_{i}=g_{i} \Delta g_{i-1} \Delta \ldots \Delta g_{1} \Delta x=$ $\left\{l_{1}, \ldots, l_{i}\right\} \Delta x$ принадлежит $X$ для каждого $i=1, \ldots, m$ и $x_{m}=z$, значит, $z \in$ $\mathscr{O}(x, X, G)$, что и требовалось доказать.

Лемма и теорема 2 доказаны. 
3.2. Несводимость к действиям группы перестановок $\mathbb{N}$. Напомним, что $S_{\infty}$ - группа всех перестановок $\mathbb{N}$, т.е. взаимно однозначных функций из $\mathbb{N}$ на $\mathbb{N}$ с суперпозицией в качестве групповой операции. Совместимая "польская" метрика на $S_{\infty}$ может быть определена через $D(x, y)=d(x, y)+d\left(x^{-1}, y^{-1}\right)$, где $d$ - обычная "польская" метрика $\mathbb{N}^{\mathbb{N}}$, т. е. $d(x, y)=2^{-m-1}$, где $m$ - наименьшее число, $x(m) \neq y(m)$.

Г. Хъёрт доказал в 1995 г., что турбулентные ОЭ борелевски несводимы к индуцированным "польскими" действиями $S_{\infty}$. Доказательство (как, например, в [6], [13]) достаточно сложно, в частности, используются некоторые теоретико-модельные факты и методы, включая анализ Скотта. Мы решили привести упрощенное доказательство, основанное на следующей теореме. Это будет все-таки довольно длинное рассуждение, так как мы даем наброски доказательств некоторых вспомогательных результатов, чтобы сделать изложение более доступным для читателя, не знакомого со специальными вопросами теории действий групп и теории моделей.

ТЕОРема 14. Любое ОЭ Е, индучированное “польским" действием и сводимое измеримой по Бәру функиией к ОЭ, индуцированному "польским" действием $S_{\infty}$, также сводимо измеримой по Бәру функцией ${ }^{13} \kappa$ одному из ОЭ $\mathrm{T}_{\gamma}$. Таким образом, по теореме 1 такое ОЭ Е не индуцируется генерически турбулентным "польским" действием.

3.3. Классифицируемость счетными структурами. Отношения изоморфизма разных классов счетных структур относятся к тем, которые индуцированы "польскими" действиями $S_{\infty}$. В самом деле, пусть $\mathscr{L}=\left\{R_{i}\right\}_{i \in I}$ - счетный реляционный язык, т. е. card $I \leqslant \aleph_{0}$, и каждое $R_{i}$ есть $m_{i}$-арный реляционный символ. $\Pi_{\text {усть }}{ }^{14} \operatorname{Mod}_{\mathscr{L}}=\prod_{i \in I} \mathscr{P}\left(\mathbb{N}^{m_{i}}\right)$ - пространство $\mathscr{L}$-структур на $\mathbb{N}$ как базовом множестве. Логическое действие $j_{\mathscr{L}}$ группы $S_{\infty}$ на $\operatorname{Mod}_{\mathscr{L}}$ определяется так: если $x=\left\{x_{i}\right\}_{i \in I} \in \operatorname{Mod}_{\mathscr{L}}$ и $g \in S_{\infty}$, то $y=j_{\mathscr{L}}(g, x)=g \cdot x=\left\{y_{i}\right\}_{i \in I} \in \operatorname{Mod}_{\mathscr{L}}$, откуда имеем

$$
\left\langle k_{1}, \ldots, k_{m_{i}}\right\rangle \in x_{i} \Longleftrightarrow\left\langle g\left(k_{1}\right), \ldots, g\left(k_{m_{i}}\right)\right\rangle \in y_{i}
$$

для всех $i \in I$ и $\left\langle k_{1}, \ldots, k_{m_{i}}\right\rangle \in \mathbb{N}^{m_{i}}$. Тогда $\left\langle\operatorname{Mod}_{\mathscr{L}} ; j_{\mathscr{L}}\right\rangle$ - "польское" $S_{\infty}$-пространство, а $j_{\mathscr{L}}$-орбиты в $\operatorname{Mod}_{\mathscr{L}}$ - это в точности классы изоморфизма $\mathscr{L}$-структур, что является причиной того, чтобы обозначить индуцированное отношение эквивалентности $\mathrm{E}_{j \mathscr{L}}^{\mathrm{Mod} \mathscr{L}}$ через $\cong \mathscr{L}$. Все ОЭ вида $\cong \mathscr{L}$ являются, конечно, аналитическими.

Г. Хъёрт [6, п. 2.38] дал определение: ОЭЕ классифицируемо счетными структурами, если есть счетный реляционньй язык $\mathscr{L}$ такой, что $\mathrm{E} \leqslant_{\mathrm{B}} \cong \mathscr{L}$.

ТЕОРема 15 [1]. Любое ОЭ, индуцированное "польским" действием $S_{\infty}$, классифицируемо счетными структурами.

\footnotetext{
13 Здесь нельзя утверждать наличие борелевской сводимости, поскольку все ОЭ, борелевски сводимые к ОЭ вида $\mathrm{T}_{\gamma}$, сами борелевские (так как все ОЭ $\mathrm{T}_{\gamma}$ борелевские), а с другой стороны, даже ОЭ вида $\cong \mathscr{L}$, вообе говоря, не борелевские (хотя и аналитические).

${ }^{14} X_{\mathscr{L}}$ часто используется вместо $\operatorname{Mod} \mathscr{L}$.
} 
Итак, все ОЭ, индуцированные “польскими” действиями $S_{\infty}$ (на самом деле, даже любой замкнутой подгруппы $S_{\infty}$ ), борелевски сводимы к весьма специальному виду действий $S_{\infty}$.

ДокАЗАтЕльство [6, п. 6.19]. Рассмотрим “польское" $S_{\infty}$-пространство $\mathbb{X}$ с базисом $\left\{U_{l}\right\}_{l \in \mathbb{N}}$ и язык $\mathscr{L}$ с символами отношений $R_{l k}$ арности $k$. Для $x \in \mathbb{X}$ определим $\vartheta(x) \in \operatorname{Mod}_{\mathscr{L}}$ так, что $\vartheta(x) \models R_{l k}\left(s_{0}, \ldots, s_{k-1}\right)$ в том случае, когда $s_{i} \neq s_{j}$ при $i<j<k$ и $g^{-1} \cdot x \in U_{l}$ всякий раз, когда $g \in S_{\infty}$ удовлетворяет $\left\langle s_{0}, \ldots, s_{k-1}\right\rangle \subset g$. Теперь $\vartheta$ сводит $\mathrm{E}_{S_{\infty}}^{\mathbb{X}} \cong \mathscr{L}$. Теорема доказана.

3.4. Редукция к счетным графам. Можно было бы ожидать, что более сложные языки $\mathscr{L}$ индуцируют более сложные ОЭ $\mathscr{L}$. Однако этого не происходит: оказывается, что одно бинарное отношение может кодировать структуры любого счетного языка. Через $\mathscr{G}$ обозначим язык (ориентированных бинарных) графов, т. е. $\mathscr{G}$ содержит единственный бинарный предикат, скажем $R(\cdot, \cdot)$.

ТЕОРЕМА 16. Если $\mathscr{L}$ - счетный реляиионный язык, то $\cong \mathscr{L}_{\mathrm{B}} \cong \mathscr{G}$.

Г. Беккер и А. Кехрис [1, п. 6.1.4] дают доказательство, основанное, в отличие от нашего рассуждения, на кодировке в терминах решеток, хотя на самом деле идея доказательства может быть одна и та же.

ДокАЗАтЕЛьство. Пусть $\mathrm{HF}(\mathbb{N})$ - множество всех наследственно конечных множеств над $\mathbb{N}$, причем элементы $\mathbb{N}$ рассматриваются как атомы, а $\varepsilon$ - соответствуюшая "принадлежность" (все $n \in \mathbb{N}$ не имеют $\varepsilon$-элементов, $\{0,1\}$ не совпадает с 2 и т. д. $)$. Через $\simeq_{\mathrm{HF}(\mathbb{N})}$ обозначим $\mathrm{HF}(\mathbb{N})$-версию $\cong \mathscr{G}$, т. е. если $P, Q \subseteq \mathrm{HF}(\mathbb{N})^{2}$, то $P \simeq_{\mathrm{HF}(\mathbb{N})} Q$ означает, что сушествует биекция $b$ множества $\mathrm{HF}(\mathbb{N})$ на себя такая, что $Q=b \cdot P=\{\langle b(s), b(t)\rangle:\langle s, t\rangle \in P\}$. Очевидно, $(\cong \mathscr{G}) \sim_{\mathrm{B}}\left(\simeq_{\mathrm{HF}(\mathbb{N})}\right)$; таким образом, следует доказать, что $\cong \mathscr{L} \leqslant_{\mathrm{B}} \simeq_{\mathrm{HF}(\mathbb{N})}$ для любого языка $\mathscr{L}$.

Действие о группы $S_{\infty}$ на $\mathrm{HF}(\mathbb{N})$ определяется так: $g \circ n=g(n)$ для $n \in \mathbb{N}$ и по $\varepsilon$-индукции $g \circ\left(\left\{a_{1}, \ldots, a_{n}\right\}\right)=\left\{g \circ a_{1}, \ldots, g \circ a_{n}\right\}$ для всех $a_{1}, \ldots, a_{n} \in \mathrm{HF}(\mathbb{N})$. Ясно, что если $g \in S_{\infty}$, то $a \mapsto g \circ a-\varepsilon$-изоморфизм $\mathrm{HF}(\mathbb{N})$.

Лемма 17. Если $X, Y \subseteq \operatorname{HF}(\mathbb{N})$ - - -транзитивные подмножества $\operatorname{HF}(\mathbb{N})$, множества $\mathbb{N} \backslash X$ и $\mathbb{N} \backslash Y$ бесконечны $и \varepsilon \uparrow X \simeq_{\mathrm{HF}(\mathbb{N})} \varepsilon \uparrow Y$, то существует перестановка $f \in S_{\infty}$ такая, что $Y=f \circ X=\{f \circ s: s \in X\}$.

ДокаЗАТЕЛЬСтво. Из предположения $\varepsilon \uparrow X \cong_{\mathrm{HF}(\mathbb{N})} \varepsilon \uparrow Y$ следует, что существует $\varepsilon$-изоморфизм $\pi: X \stackrel{\text { на }}{\rightarrow} Y$. Легко видеть, что $\pi \uparrow(X \cap \mathbb{N})-$ биекция $X_{0}=X \cap \mathbb{N}$ на $Y_{0}=Y \cap \mathbb{N}$, значит, имеется $f \in S_{\infty}$ такое, что $f \uparrow X_{0}=\pi \uparrow X_{0}$, и тогда выполнено $f \circ s=\pi(s)$ для каждого $s \in X$. Лемма доказана.

Возвращаясь к доказательству теоремы 16 , сначала покажем, что имеет место $\cong_{\mathscr{G}(m)} \leqslant_{\mathrm{B}} \simeq_{\mathrm{HF}(\mathbb{N})}$ для каждого $m \geqslant 3$, где $\mathscr{G}(m)$ - язык с единственным $m$-арным предикатом. Заметим, что $\left\langle i_{1}, \ldots, i_{m}\right\rangle \in \mathrm{HF}(\mathbb{N})$ всякий раз, когда $i_{1}, \ldots, i_{m} \in \mathbb{N}$. Положим $\Theta(x)=\{\vartheta(s): s \in x\}$ для каждого $x \in \operatorname{Mod}_{\mathscr{G}(m)}=\mathscr{P}\left(\mathbb{N}^{m}\right)$, где $\vartheta(s)=$ $\mathrm{TC}_{\varepsilon}\left(\left\{\left\langle 2 i_{1}, \ldots, 2 i_{m}\right\rangle\right\}\right)$ для всех $s=\left\langle i_{1}, \ldots, i_{m}\right\rangle \in \mathbb{N}^{m}$, и, наконец, для $X \subseteq \mathrm{HF}(\mathbb{N})$ пусть $\mathrm{TC}_{\varepsilon}(X)$ - наименьшее из тех $\varepsilon$-транзитивных множеств $T \subseteq \operatorname{HF}(\mathbb{N})$, которые удовлетворяют $X \subseteq T$. Из леммы 17 легко следует, что $x \cong \mathscr{G}(m) y$ равносильно $\varepsilon \uparrow \Theta(x) \simeq_{\mathrm{HF}(\mathbb{N})} \varepsilon \uparrow \Theta(y)$, что завершает доказательство соотношения $\cong_{\mathscr{G}(m)} \leqslant_{\mathrm{B}} \simeq_{\mathrm{HF}(\mathbb{N})}$. 
Остается вывести $\cong_{\mathscr{L}^{\prime}} \leqslant_{\mathrm{B}} \simeq_{\mathrm{HF}(\mathbb{N})}$, где $\mathscr{L}^{\prime}$ - язык с бесконечным числом бинарных предикатов. В этом случае $\operatorname{Mod}_{\mathscr{L}^{\prime}}=\mathscr{P}\left(\mathbb{N}^{2}\right)^{\mathbb{N}}$, так что можно предполо-

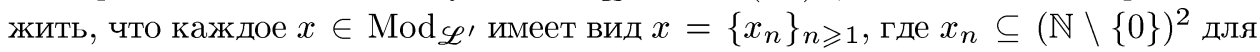
всех $n$. Пусть $\Theta(x)=\left\{s_{n}(k, l): n \geqslant 1 \wedge\langle k, l\rangle \in x_{n}\right\}$ для каждого такого $x$, где $s_{n}(k, l)=\mathrm{TC}_{\varepsilon}(\{\{\ldots\{\langle k, l\rangle\} \ldots\}, 0\})$ с $n+2$ парами скобок $\{$,$\} . Тогда \Theta$ есть непрерывная редукция $\cong_{\mathscr{L}^{\prime} \mathrm{K}} \simeq_{\mathrm{HF}(\mathbb{N})}$. Теорема доказана.

3.5. Доказательство теоремы 14. Доказательство (являющееся вариантом рассуждения, приведенного в [4]) основано на анализе Скотта.

Определим семейство борелевских бинарных отношений $\equiv_{s t}^{\alpha}$ на $\mathscr{P}\left(\mathbb{N}^{2}\right)$, где $\alpha<\omega_{1}$ и $s, t \in \mathbb{N}<\omega$, следуюшим образом:

(i) $A \equiv_{s t}^{0} B$, когда $A\left(s_{i}, s_{j}\right) \Longleftrightarrow B\left(t_{i}, t_{j}\right)$ для всех $i, j<\operatorname{lh} s=\operatorname{lh} t$;

(ii) $A \equiv_{s t}^{\alpha+1} B$, когда $\forall k \exists l\left(A \equiv_{s^{\wedge} k, t^{\wedge} l}^{\alpha} B\right)$ и $\forall l \exists k\left(A \equiv_{s^{\wedge} k, t^{\wedge} l}^{\alpha} B\right)$;

(iii) если $\lambda<\omega_{1}$ пределен, то $A \equiv_{s t}^{\lambda} B$, когда $A \equiv_{s t}^{\alpha} B$ для всех $\alpha<\lambda$.

Определим $\langle s, A\rangle \equiv^{\alpha}\langle t, B\rangle$, если $A \equiv_{s t}^{\alpha} B$; тогда, индукцией по $\alpha$, каждое $\equiv^{\alpha}$ является борелевским ОЭ на $\mathbb{N}<\omega \times \mathscr{P}\left(\mathbb{N}^{2}\right)$ и $\equiv{ }^{\beta} \subseteq \equiv^{\alpha}$ при $\alpha<\beta$.

Рассмотрим $\mathrm{O}$ Э $\mathrm{E}=\mathrm{E}_{\mathbb{G}}^{\mathbb{X}}$, индуцированное "польским" действием "польской" группы $\mathbb{G}$ на "польском" пространстве $\mathbb{X}$, которое сводится к "польскому" действию $S_{\infty}$ при помощи измеримой по Бэру функции. Согласно теоремам 15,16 и предложению 3 существует измеримая по Бэру редукция $\vartheta: \mathbb{X} \rightarrow \mathscr{P}\left(\mathbb{N}^{2}\right)$ отношения $\mathrm{E}$ к $\cong \mathscr{g}$. Она непрерывна на плотном $\mathbf{G}_{\delta}$-множестве $D_{0} \subseteq \mathbb{X}$. Напомним, что для $A, B \subseteq \mathbb{N}^{2}$ соотношение $A \cong \mathscr{G} \quad B$ означает, что найдется функция $f \in S_{\infty}$ такая, что $A(k, l) \Longleftrightarrow B(f(k), f(l))$ для всех $k, l$. Индукцией по $\alpha$ мы легко получаем $\cong_{\mathscr{G}} \subseteq \equiv_{s t}^{\alpha}$, где $t=f \circ s$, в частности, $\cong_{\mathscr{g}} \subseteq \equiv_{\Lambda \Lambda}^{\alpha}$, где $\Lambda-$ пустая последовательность. Поскольку $\vartheta$ является редукцией, эквивалентность $x \mathrm{E} y \Longleftrightarrow \vartheta(x) \cong \mathscr{G} \vartheta(y)$ выполнена для всех $x, y$. Наша цель - найти плотное $\mathbf{G}_{\delta}$-множество $D \subseteq D_{0}$ и ординал $\alpha<\omega_{1}$ такие, что

$(*)$ импликация $x \not E y \Longrightarrow \vartheta(x) \#_{\Lambda \Lambda}^{\alpha} \vartheta(y)$ верна для всех $x, y \in D$.

Для этого фиксируем счетную транзитивную модель $\mathfrak{M}$ теории ZFHC (см. выше). Предполагается, что пространство $\mathbb{X}$, группа $\mathbb{G}$, ее действие на $\mathbb{X}$, множество $D_{0}$ и функция $\vartheta \uparrow D_{0}$ кодированы в $\mathfrak{M}$ в том смысле, как указано в доказательстве леммы 9 . Покажем, что множество $D$ всех генерических по Коэну над $\mathfrak{M}$ точек $\mathbb{X}$ (т. е. плотное $\mathbf{G}_{\delta}$-подмножество $\mathbb{X}$, оно включено в $D_{0}$ ) удовлетворяет $(*)$.

Пусть $x, y \in D$. Предположим сначала, что пара $\langle x, y\rangle$ является генерической по Коэну над $\mathfrak{M}$. По выбору $\vartheta$, если $x \mathbb{Z} y$, мы имеем $\vartheta(x) \neq \mathscr{q} \vartheta(y)$, значит, по теореме абсолютности Мостовского соотношение $\vartheta(x) \neq \mathscr{G} \vartheta(y)$ выполнено в $\mathfrak{M}[x, y]$. Следовательно, рассуждая релятивизованно к модели $\mathfrak{M}[x, y]$ (это все еще модель ZFHC, см. п. 2.3), находим ординал $\alpha \in \operatorname{Ord}^{\mathfrak{M}}=\operatorname{Ord}^{\mathfrak{M}[x, y]}, \vartheta(x) \not_{\Lambda \Lambda}^{\alpha} \vartheta(y)$. Более того, поскольку форсинг Коэна удовлетворяет ССС (условию счетности антицепей), сушествует ординал $\alpha \in \mathfrak{M}$ такой, что $\vartheta(x) \not_{\Lambda \Lambda}^{\alpha} \vartheta(y)$ для всех генерических по Коэну над $\mathfrak{M}$ пар $\langle x, y\rangle \in D^{2}, x \notin Z y$. Остается показать, что это также выполнено для всех пар $x, y \in D, x \notin Z y$, которые не образуют генерическую по Коэну пару.

Рассмотрим генерический по Коэну над $\mathfrak{M}[x, y]$ элемент $g \in \mathbb{G}$. Легко видеть, что $z=g \cdot x \in \mathbb{X}$ - генерическая по Коэну над $\mathfrak{M}[x, y]$ точка (поскольку действие непрерывно), более того, $x \mathrm{E} z$, значит, $y \mathbb{Z} z$. Однако $y$ генерично по Коэну над $\mathfrak{M}$, 
а $z$ генерично над $\mathfrak{M}[y]$, значит, пара $\langle y, z\rangle$ генерическая по Коэну над $\mathfrak{M}$, таким образом, $\vartheta(z) \not_{\Lambda \Lambda}^{\alpha} \vartheta(y)$ по доказанному вьше. С другой стороны, $\vartheta(x) \equiv_{\Lambda \Lambda}^{\alpha} \vartheta(z)$ выполнено, поскольку $x$ Е $z$. Имеем в конце концов $\vartheta(x) \not_{\Lambda \Lambda}^{\alpha} \vartheta(y)$, что и требуется в $(*)$.

Итак, $x \mathrm{E} y \Longleftrightarrow \vartheta(x) \equiv_{\Lambda \Lambda}^{\alpha} \vartheta(y)$ для всех $x, y \in D$. Ясно, что $\vartheta$ можно переопределить на дополнении к $D$ в $\mathbb{X}$ так, что эквивалентность выполнится для всех $x, y \in \mathbb{X}$, иными словами, измененная $\vartheta$ есть (так как $\vartheta \uparrow D$ непрерывна, а $D$ плотное $\mathbf{G}_{\delta}$-множество) измеримая по Бэру редукция $\mathrm{E}_{\mathrm{K}} \equiv_{\Lambda \Lambda}^{\alpha}$.

Следуюший результат завершает доказательство теоремы.

ПРЕДЛОЖЕНИЕ 18. Каждое ОЭ ${ }^{\alpha}$ борелевски сводимо к одному из $\mathrm{T}_{\gamma}$.

ДокАЗАТЕЛЬСТво. Вьполнено $\equiv^{0} \leqslant{ }_{\mathrm{B}} \mathrm{T}_{0}$, так как $\equiv^{0}$ имеет счетно много классов эквивалентности и все они открыто-замкнуты. Чтобы провести шаг $\alpha \mapsto \alpha+1$, заметим, что отображение $\langle s, A\rangle \mapsto\left\{\left\langle s^{\wedge} k, A\right\rangle\right\}_{k \in \mathbb{N}}$ есть борелевская редукция $\equiv^{\alpha+1} \mathrm{\kappa}\left(\equiv^{\alpha}\right)^{\infty}$. Пусть $\lambda=\left\{\alpha_{n}: n \in \mathbb{N}\right\}$ - предельньй ординал и $\mathrm{R}=\bigvee_{n \in \mathbb{N}} \equiv^{\alpha_{n}}$, т. е. $\mathrm{R}$ есть ОЭ на $\mathbb{N} \times \mathbb{N}<\omega \times \mathscr{P}\left(\mathbb{N}^{2}\right)$, определенное так, что $\langle m, s, A\rangle \mathrm{R}\langle n, t, B\rangle$, когда $m=n$ и $A \equiv_{s t}^{\alpha_{m}} B$. Однако отображение $\langle s, A\rangle \mapsto\{\langle m, s, A\rangle\}_{m \in \mathbb{N}}$ является борелевской редукцией $\equiv^{\lambda}{ }_{\mathrm{K}} \mathrm{R}^{\infty}$.

Теорема 14 доказана.

\section{§4. Приколотые ОЭ и несводимость $\mathrm{T}_{2}$}

Этот параграф̆ включает теорему, согласно которой ОЭ $\mathrm{T}_{2}$ (равенство счетных множеств вещественных чисел) борелевски не сводится к ОЭ из семейства приколотых $\mathrm{O}$ Э, которое включает, например, непрерывные действия CLI-групп, некоторые не обязательно "полишабельные" идеалы, ОЭ с классами эквивалентности в $\mathbf{G}_{\delta \sigma}$, и замкнуто относительно произведений Фубини по модулю Fin. Определение этого семейства основано на свойстве скорее метаматематического характера, которое мы взяли из статьи [5].

4.1. Приколотые ОЭ. Прежде всего, договоримся, что если $X$ - аналитическое множество в универсуме всех множеств $\mathbb{V}$ (в частности, $X$ может быть борелевским) и рассматривается генерическое расширение $\mathbb{V}^{+}$универсума $\mathbb{V}$, то через $X^{\sharp}$ будет обозначаться результат той последовательности операций, которая заключена в определении $X$, но примененной в $\mathbb{V}^{+}$. Это определение корректно по теореме абсолютности Шенфилда, и легко видеть, что $X=X^{\sharp} \cap \mathbb{V}$.

Например, если в универсуме $\mathbb{V}$ отношение $\mathbb{E}$ есть аналитическое $О Э$ на “польском" пространстве $\mathbb{X}$, то, по теореме абсолютности Шенфилда, $\mathbb{E}^{\sharp}$ - аналитическое ОЭ на $\mathbb{X}^{\sharp}$. Если $x \in \mathbb{X}$ (значит, $x \in \mathbb{V}$ ), то Е-класс $[x]_{\mathrm{E}} \subseteq \mathbb{X}$ точки $x$ (определенньй в $\mathbb{V})$ включен в единственный $\mathbb{E}^{\sharp}$-класс $[x]_{E^{\sharp}} \subseteq \mathbb{X}^{\sharp}\left(\right.$ в $\left.\mathbb{V}^{+}\right)$. Классы вида $[x]_{\mathbb{E}^{\sharp}}$, $x \in \mathbb{X}$, принадлежат к более широкой, вообше говоря, категории $\mathbb{E}^{\sharp}$-классов, допускаюших описание с точки зрения универсума $\mathbb{V}$.

ОПРЕДЕЛЕНИЕ 19. Допустим, что $\mathbb{P}$ - форсинг в $\mathbb{V}$. Виртуальныц Е-классом называется всякий $\mathbb{P}$-терм $\boldsymbol{\xi}$ такой, что $\mathbb{P}$ вынуждает $\boldsymbol{\xi} \in \mathbb{X}^{\sharp}$ и $\mathbb{P} \times \mathbb{P}$ вынуждает 
$\boldsymbol{\xi}_{\text {л }} \mathrm{E}^{\sharp} \boldsymbol{\xi}_{\text {пр }}{ }^{15}$. Виртуальный класс называется приколотым, если существует в $\mathbb{V}$ точка $x \in \mathbb{X}$, которая "прикалывает" его в том смысле, что $\mathbb{P}$ вынуждает $x \mathrm{E}^{\sharp} \boldsymbol{\xi}$. Наконец, аналитическое ОЭ $\mathrm{E}$ приколото, если для любого форсинга $\mathbb{P} \in \mathbb{V}$ все виртуальные Е-классы приколоты.

Если $\boldsymbol{\xi}$ - виртуальный Е-класс, то в любом расширении $\mathbb{V}^{+}$универсума $\mathbb{V}$ выполнено следуюшее: если $U$ и $V$-генерические подмножества $\mathbb{P}$, то точки $x=\boldsymbol{\xi}[U]$ и $y=\boldsymbol{\xi}[V]$ принадлежат $\mathbb{X}^{\sharp}$ и удовлетворяют $x \mathbb{E}^{\sharp} y$, следовательно, $\boldsymbol{\xi}$ индуцирует $\mathbb{E}^{\sharp}$-класс в расширении. Если $\boldsymbol{\xi}$ приколот, то этот класс содержит элемент из исходного универсума $\mathbb{V}$, другими словами, приколотые виртуальные классы индуцируют классы $\mathbb{E}^{\sharp}$-эквивалентности вида $[x]_{\mathbb{E}^{\sharp}}, x \in \mathbb{V}$, в расширениях $\mathbb{V}$.

Докажем ниже, что $\mathrm{T}_{2}$ не приколото, более того, $\mathrm{T}_{2}$ борелевски не сводится ни к какому приколотому аналитическому ОЭ. В дополнение дадим упрощенное доказательство теоремы Хъёрта о том, что непрерывные действия "польских" CLI-групп не индуцируют приколотые ОЭ, представим семейство приколотых ОЭ, порожденных $\mathbf{F}_{\sigma \delta}$-идеалами, докажем, что борелевские ОЭ с классами эквивалентности типа $\mathbf{G}_{\delta \sigma}$ приколоты, и докажем, что класс всех приколотых аналитических ОЭ замкнут относительно произведения Фубини по модулю Fin.

4.2. Приколотые ОЭ и $\mathrm{T}_{2}$. Напомним, что, с точностью до $\sim_{\mathrm{B}}$, отношение $\mathrm{T}_{2}$ есть ОЭ на $\left(2^{\mathbb{N}}\right)^{\mathbb{N}}$, определенное так: $x \mathrm{~T}_{2} y$, когда $\operatorname{ran} x=\operatorname{ran} y$.

ЛЕмма 20. Отношение $\mathrm{T}_{2}$ не приколото. Если $\mathrm{E}, \mathrm{F}$ - аналитические ОЭ, $\mathrm{E} \leqslant_{\mathrm{B}} \mathrm{F}$ и $\mathrm{F}$ приколото, то $\mathrm{E}$ также приколото. Следовательно, $\mathrm{T}_{2}$ борелевски не сводится ни к одному приколотому аналитическому ОЭ.

ДокАЗАтЕльство. Для доказательства того, что $\mathrm{T}_{2}$ не приколото, рассмотрим в $\mathbb{V}$ форсинг $\mathbb{P}=\operatorname{Coll}\left(\mathbb{N}, 2^{\mathbb{N}}\right)$, порождающий генерическую функцию $f: \mathbb{N} \stackrel{\text { на }}{\rightarrow} 2^{\mathbb{N}}$ $\left(\mathbb{P}\right.$ состоит из всех функций $p: u \rightarrow 2^{\mathbb{N}}$, где $u \subseteq \mathbb{N}$ конечно). $\mathbb{P}$-терм $\boldsymbol{\xi}$ для $\operatorname{ran} f=$ $\{f(n): n \in \mathbb{N}\}$ является виртуальньм $\mathbf{T}_{2}$-классом, но он не приколот, поскольку $2^{\mathbb{N}}$ несчетно в исходном универсуме $\mathbb{V}$.

Допустим, что $\vartheta: \mathbb{X} \rightarrow \mathbb{Y}$ в $\mathbb{V}$ является борелевской редукцией $\mathrm{E}$ к $\mathrm{F}$, где $\mathbb{X}=$ $\operatorname{dom} E$ и $\mathbb{Y}=\operatorname{dom} F$. Можно предполагать, что $\mathbb{X}=\mathbb{Y}=2^{\mathbb{N}}$. Рассмотрим форсинг $\mathbb{P}$ и $\mathbb{P}$-терм $\boldsymbol{\xi}$, являюшийся виртуальным $\mathrm{E}$-классом. По теореме абсолютности Шенфилда $\vartheta^{\sharp}$ будет редукцией $E^{\sharp}$ к $F^{\sharp}$ в любом расширении $\mathbb{V}$, значит, $\mathbb{P}$-терм $\sigma$ для $\vartheta^{\sharp}(\boldsymbol{\xi})$ также есть виртуальный $\mathrm{F}$-класс. Поскольку $\mathrm{F}$ приколото, найдется $y \in \mathbb{Y}$ такое, что $\mathbb{P}$ вынуждает $y \mathrm{~F}^{\sharp} \boldsymbol{\sigma}$. Заметим, что в $\mathbb{P}$-генерическом расширении выполнено $y \mathrm{~F}^{\sharp} \vartheta^{\sharp}(x)$ для некоторого $x \in \mathbb{X}^{\sharp}$, значит, по теореме Шенфилда в исходном универсуме имеется $x \in \mathbb{X}$ такое, что $y \mathrm{~F} \vartheta(x)$. Легко видеть, что $\mathbb{P}$ вынуждает $x \mathrm{E}^{\sharp} \boldsymbol{\xi}$. Лемма доказана.

4.3. Произведения Фубини приколотых ОЭ. Напомним, что произведение Фубини $\mathrm{E}=\prod_{k \in \mathbb{N}} \mathrm{E}_{k} /$ Fin данных ОЭ $\mathrm{E}_{k}$ на $\mathbb{X}_{k}$ по модулю Fin есть ОЭ на $\mathbb{X}=\prod_{k} \mathbb{X}_{k}$, определенное так, что выполнено $x \mathrm{E} y$, когда выполнено $x(k) \mathrm{E}_{k} y(k)$ для всех, кроме конечного числа, индексов $k$.

\footnotetext{
${ }^{15} \boldsymbol{\xi}_{\text {л }}$ и $\boldsymbol{\xi}_{\text {пр }}$ - это $(\mathbb{P} \times \mathbb{P})$-термы, означающие терм $\boldsymbol{\xi}$, ассоциированный с соответственно левым и правым факторами $\mathbb{P}$ в произведении форсингов, формально, $\boldsymbol{\xi}_{\text {л }}[U \times V]=\boldsymbol{\xi}[U]$ и $\boldsymbol{\xi}_{\text {пр }}[U \times V]=\boldsymbol{\xi}[V]$ для любого $(\mathbb{P} \times \mathbb{P})$-генерического множества $U \times V$, где, скажем, $\boldsymbol{\xi}[U]-$ интерпретация терма $\boldsymbol{\xi}$ через генерическое множество $U$.
} 
ЛЕмма 21. Семейство всех аналитических приколотых ОЭ замкнуто относительно произведений Фубини по модулю Fin.

ДокАЗАтЕльство. Допустим, что аналитические ОЭ $\mathrm{E}_{k}$ на “польских" пространствах $\mathbb{X}_{k}$ приколоты. Докажем, что их произведение Фубини, т. е. ОЭ $\mathrm{E}=$ $\prod_{k \in \mathbb{N}} \mathrm{E}_{k} /$ Fin на $\mathbb{X}=\prod_{k} \mathbb{X}_{k}$, также приколото. Рассмотрим форсинг $\mathbb{P}$ и $\mathbb{P}$-терм $\boldsymbol{\xi}$, являющийся виртуальным Е-классом. Сушествуют число $k_{0}$ и "условия" $p, q \in$ $\mathbb{P}$ такие, что $\langle p, q\rangle(\mathbb{P} \times \mathbb{P})$-вынуждает $\boldsymbol{\xi}_{\text {л }}(k) \mathrm{E}_{k}^{\sharp} \boldsymbol{\xi}_{\text {пр }}(k)$ для всех $k \geqslant k_{0}$. Поскольку все $\mathrm{E}_{k}$ суть ОЭ, мы заключаем, что “условие" $\langle p, p\rangle$ также вынуждает $\boldsymbol{\xi}_{\text {л }}(k) \mathrm{E}_{k}^{\sharp} \boldsymbol{\xi}_{\text {пр }}(k)$ для всех $k \geqslant k_{0}$. Следовательно, так как $\mathrm{E}_{k}$ приколоты, существует в $\mathbb{V}$ последовательность точек $x_{k} \in \mathbb{X}_{k}$ таких, что $p \mathbb{P}$-вынуждает $x_{k} \mathrm{E}_{k}^{\sharp} \boldsymbol{\xi}(k)$ для всех $k \geqslant k_{0}$. Рассмотрим $x \in \mathbb{X}$ такое, что $x(k)=x_{k}$ для всех $k \geqslant k_{0}$ (значения $x(k) \in \mathbb{X}_{k}$ при $k<k_{0}$ могут быть любые). Тогда $p$, очевидно, $\mathbb{P}$-вынуждает $x \mathrm{E}^{\sharp} \boldsymbol{\xi}$.

Остается показать, что каждое $q \in \mathbb{P}$ вынуждает $x \mathrm{E}^{\sharp} \boldsymbol{\xi}$. Пусть, напротив, некоторое $q \in \mathbb{P}$ вынуждает то, что $x \mathbb{E}^{\sharp} \boldsymbol{\xi}$ не имеет места. Рассмотрим пару $\langle p, q\rangle$ как

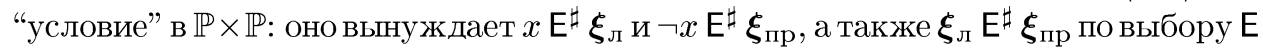
и $\boldsymbol{\xi}$. Противоречие. Лемма доказана.

4.4. Инвариантные слева действия и приколотые ОЭ. Напомним, что “польская" группа $\mathbb{G}$ назьвается CLI-груnпой (complete left-invariant), если она допускает совместимую полную инвариантную слева метрику. Тогда $\mathbb{G}$ также допускает и совместимую полную инвариантную справа метрику, что и будет практически использовано.

ТЕОРема 22 [5]. Любое ОЭ $\mathrm{E}=\mathrm{E}_{\mathbb{G}}^{\mathbb{X}}$, индуцированное "польским" действием CLI-группье $\mathbb{G}$ на "польском" пространстве $\mathbb{X}$, приколото, значит, $\mathrm{T}_{2}$ борелевски не сводится $\kappa \mathrm{E}$.

ДоказАтельство. Рассмотрим форсинг $\mathbb{P}$ и виртуальный Е-класс $\boldsymbol{\xi}$. Через $\leqslant$ обозначается частичный порядок на $\mathbb{P}$; как обычно, $p \leqslant q$ означает, что $p$ - более сильное "условие". Фиксируем совместимую полную инвариантную справа метрику $\rho$ на $\mathbb{G}$. Для $\varepsilon>0$ положим $G_{\varepsilon}=\left\{g \in \mathbb{G}: \rho\left(g, 1_{\mathbb{G}}\right)<\varepsilon\right\}$. Скажем, что $q \in \mathbb{P}$ имеет размер $\leqslant \varepsilon$, если $\langle q, q\rangle(\mathbb{P} \times \mathbb{P})$-вынуждает существование $g \in G_{\varepsilon}^{\sharp}$ такого, что $\boldsymbol{\xi}_{\text {л }}=g \cdot \boldsymbol{\xi}_{\text {пр }}$.

Лемма 23. Если $q \in \mathbb{P} u \varepsilon>0$, то существует "условие" $r \in \mathbb{P}, \quad r \leqslant q$, размера $\leqslant \varepsilon$.

ДокАЗАТЕльство. Пусть, напротив, для каждого $r \in \mathbb{P}, r \leqslant q$, имеется пара “условий" $r^{\prime}, r^{\prime \prime} \in \mathbb{P}$, более сильных, чем $r$, и таких, что "условие" $\left\langle r^{\prime}, r^{\prime \prime}\right\rangle$ $(\mathbb{P} \times \mathbb{P})$-вынуждает несуществование $g \in G_{\varepsilon}^{\sharp}$ такого, что $\boldsymbol{\xi}_{л}=g \cdot \boldsymbol{\xi}_{\text {пр }}$. Применяя в таком генерическом расширении $\mathbb{V}^{+}$универсума $\mathbb{V}$, где $\mathscr{P}(\mathbb{P}) \cap \mathbb{V}$ счетно, обычную расщепляющую конструкцию, находим несчетное множество $\mathscr{U}$ генерических множеств $U \subseteq \mathbb{P}, q \in U$, такое, что все пары $\langle U, V\rangle$ из $U \neq V$ в $\mathscr{U}$ суть $(\mathbb{P} \times \mathbb{P})$-генерические (над $\mathbb{V})$, значит, нет $g \in G_{\varepsilon}^{\sharp}$ такого, что $\boldsymbol{\xi}[U]=g \cdot \boldsymbol{\xi}[V]{ }^{16}$.

Рассмотрим $U_{0} \in \mathscr{U}$. Каждому $U \in \mathscr{U}$ можно сопоставить в $\mathbb{V}^{+}$элемент $g_{U} \in G^{\sharp}$ такой, что $\boldsymbol{\xi}[U]=g_{U} \cdot \boldsymbol{\xi}\left[U_{0}\right]$, тогда $g_{U} \notin G_{\varepsilon}^{\sharp}$ (см. выше). Более того,

\footnotetext{
${ }^{16} \boldsymbol{\xi}[U]$ есть интерпретация $\mathbb{P}$-терма $\boldsymbol{\xi}$ посредством генерического множества $U$.
} 
мы имеем $g_{V} g_{U}^{-1} \cdot \boldsymbol{\xi}[U]=\boldsymbol{\xi}[V]$ для всех $U, V \in \mathscr{U}$, значит, $g_{V} g_{U}^{-1} \notin G_{\varepsilon}^{\sharp}$ при $U \neq V$, что влечет $\rho\left(g_{U}, g_{V}\right) \geqslant \varepsilon$ согласно инвариантности метрики справа. Но это противоречит сепарабельности $G$. Лемма доказана.

Возвращаемся к доказательству теоремы 22. Предположим, что, напротив, "условие" $p \in \mathbb{P}$ вынуждает отсутствие точек $x \in \mathbb{X}$ (в исходном универсуме $\mathbb{V}$ ) таких, что $x \mathrm{E}^{\sharp} \boldsymbol{\xi}$. Согласно лемме 23 сушествуют в $\mathbb{V}$ последовательности "условий" $p_{n} \in \mathbb{P}$ размера $\leqslant 2^{-n}$ и замкнутые множества $X_{n} \subseteq \mathbb{X} \mathbb{X}$-диаметра $\leqslant 2^{-n}$ такие, что $p_{0} \leqslant p, p_{n+1} \leqslant p_{n}, X_{n+1} \subseteq X_{n}$ и $p_{n}$ вынуждает $\boldsymbol{\xi} \in X_{n}^{\sharp}$ для каждого $n$. Пусть $x$ - общая точка множеств $X_{n}$ в $\mathbb{V}$. Утверждается, что $p_{0}$ вынуждает $x \mathrm{E}^{\sharp} \boldsymbol{\xi}$.

В самом деле, иначе некоторое $q \in \mathbb{P}, q \leqslant p_{0}$, вынуждает $\neg x \mathbb{E}^{\sharp} \boldsymbol{\xi}$. Рассмотрим расширение $\mathbb{V}^{+}$универсума $\mathbb{V}$, содержашее для каждого $n$ генерическое множество $U_{n} \subseteq \mathbb{P}, p_{n} \in U_{n}$, такое, что каждая пара $\left\langle U_{n}, U_{n+1}\right\rangle$ - $(\mathbb{P} \times \mathbb{P})$-генерическая $($ над $\mathbb{V})$ и, кроме того, $q \in U_{0}$. Пусть $x_{n}=\boldsymbol{\xi}\left[U_{n}\right]$ (элемент $\left.\mathbb{X}^{\sharp}\right)$; тогда $\left\{x_{n}\right\} \rightarrow x$. Для каждого $n$ как $U_{n}$, так и $U_{n+1}$ содержат $p_{n}$, значит, поскольку $p_{n}$ имеет размер $\leqslant 2^{-n-1}$, сушествует $g_{n+1} \in \mathbb{G}_{\varepsilon}^{\sharp}$ такое, что $x_{n+1}=g_{n+1} x_{n}$. Тогда $x_{n}=h_{n} \cdot x_{0}$, где $h_{n}=g_{n} \ldots g_{1}$. Однако $\rho\left(h_{n}, h_{n-1}\right)=\rho\left(g_{n}, 1_{\mathbb{G}}\right) \leqslant 2^{-n+1}$ по правой инвариантности метрики $\rho$, значит, $\left\{h_{n}\right\}_{n \in \mathbb{N}}-$ последовательность Коши в $\mathbb{G}^{\sharp}$. Через $h=\lim _{n \rightarrow \infty} h_{n} \in \mathbb{G}^{\sharp}$ обозначим ее предел. Поскольку рассматриваемое действие непрерьвно, $x=\lim _{n} x_{n}=h \cdot x_{0}$. Следовательно, мы имеем $x \mathrm{E}^{\sharp} x_{0}$ в $\mathbb{V}^{+}$, значит, также и в $\mathbb{V}\left[U_{0}\right]$. Однако $x_{0}=\boldsymbol{\xi}\left[U_{0}\right]$, в то время как $q \in U_{0}$ вынуждает $\neg x \mathrm{E}^{\sharp} \boldsymbol{\xi}$. Противоречие.

Итак, $p_{0} \mathbb{P}$-вьнуждает $x \mathrm{E}^{\sharp} \boldsymbol{\xi}$. Тогда все $r \in \mathbb{P}$ вынуждают $x \mathrm{E}^{\sharp} \boldsymbol{\xi}$. В самом деле, если $r \in \mathbb{P}$ вынуждает $\neg x \mathrm{E}^{\sharp} \boldsymbol{\xi}$, то пара $\left\langle p_{0}, r\right\rangle(\mathbb{P} \times \mathbb{P})$-вынуждает $x \mathrm{E}^{\sharp} \boldsymbol{\xi}_{\text {л }}$ и $\neg x \mathbb{E}^{\sharp} \boldsymbol{\xi}_{\text {пр }}$, что противоречит тому, что $(\mathbb{P} \times \mathbb{P})$-вынуждает $\boldsymbol{\xi}_{\text {л }} \mathbb{E}^{\sharp} \boldsymbol{\xi}_{\text {пр }}$. Теорема 22 доказана.

4.5. ОЭ $\mathbf{c ~ G}_{\delta \sigma}$-классами. Рассмотрим не приколотое ОЭ $\mathrm{T}_{2}$ класса $\mathbf{F}_{\sigma \delta}$. Следующая теорема показывает, что это наиболее простой класс не приколотых борелевских ОЭ.

ТЕОРЕма 24. Каждде борелевское ОЭ Е, все классы әквивалентности которого - множества $\mathbf{G}_{\delta \sigma}$, является приколотым.

ДокАЗАтельство (основано на идее, сообщенной авторам Хъёртом). Можно предполагать, что dom $\mathrm{E}=\mathbb{N}^{\mathbb{N}}$. Из теоремы Луво [14] следует, что существует борелевская функция $\gamma$, определенная на $\mathbb{N}^{\mathbb{N}}$ так, что $\gamma(x)$ есть $\mathbf{G}_{\delta \sigma}$-код $[x]_{\mathrm{E}}$ для каждого $x \in \mathbb{N}^{\mathbb{N}}$, т. е., например, $\gamma(x) \subseteq \mathbb{N}^{2} \times \mathbb{N}<\omega$ и

$$
[x]_{\mathrm{E}}=\bigcup_{i} \bigcap_{j} \bigcup_{\langle i, j, s\rangle \in \gamma(x)} B_{s}, \quad B_{s}=\left\{a \in \mathbb{N}^{\mathbb{N}}: s \subset a\right\}, \quad s \in \mathbb{N}<\omega
$$

Рассмотрим форсинг $\mathbb{P}=\langle\mathbb{P} ; \leqslant\rangle$ и виртуальный Е-класс $\boldsymbol{\xi}$, таким образом, $\mathbb{P} \times \mathbb{P}$ вынуждает $\boldsymbol{\xi}_{\text {л }} \mathbb{E}^{\sharp} \boldsymbol{\xi}_{\text {пр }}$, значит, имеются число $i_{0}$ и “условие" $\left\langle p_{0}, q_{0}\right\rangle \in \mathbb{P} \times \mathbb{P}$, вынуждающее $\boldsymbol{\xi}_{\text {л }} \in \vartheta^{\sharp}\left(\boldsymbol{\xi}_{\text {пр }}\right)$, где $\vartheta(x)=\bigcap_{j} \bigcup_{\left\langle i_{0}, j, s\right\rangle \in \gamma(x)} B_{s}$ для $x \in \mathbb{N}^{\mathbb{N}}$.

Главной идеей доказательства является замена $\mathbb{P}$ форсингом Коэна. Обозначим через $\mathbb{S}$ множество всех $s \in \mathbb{N}<\omega$ таких, что $p_{0}$ не $\mathbb{P}$-вынуждает $s \not \subset \boldsymbol{\xi}$. Рассмотрим $\mathbb{S}$ как форсинг, и $s \subseteq t$ (т.е. $t$ продолжает $s$ ) означает, что $t$ - более сильное 
"условие"; пустая последовательность $\Lambda$ является слабейшим "условием" в $\mathbb{S}$. Ecли $s \in \mathbb{S}$, то сушествует по меньшей мере одно $n$ такое, что $s^{\wedge} n \in \mathbb{S}$, следовательно, $\mathbb{S}$ вынуждает элемент $\mathbb{N}^{\mathbb{N}}, \mathbb{S}$-имя которого будет а.

Лемма 25. Пара $\left\langle\Lambda, q_{0}\right\rangle(\mathbb{S} \times \mathbb{P})$-вынуждает $\mathbf{a} \in \vartheta^{\sharp}(\boldsymbol{\xi})$.

ДокАЗАТЕльСтво. В противном случае некоторое "условие" $\left\langle s_{0}, q\right\rangle \in \mathbb{S} \times \mathbb{P}$, $q \leqslant q_{0}$, вынуждает $\mathbf{a} \notin \vartheta^{\sharp}(\boldsymbol{\xi})$. По определению $\vartheta$ можно предполагать, что

$$
\left\langle s_{0}, q\right\rangle(\mathbb{S} \times \mathbb{P}) \text {-вынуждает } \quad \neg \exists s\left(\left\langle i_{0}, j_{0}, s\right\rangle \in \gamma(\boldsymbol{\xi}) \wedge s \subset \mathbf{a}\right)
$$

для некоторого $j_{0}$. Так как $s_{0} \in \mathbb{S}$, имеется "условие" $p^{\prime} \in \mathbb{P}, p^{\prime} \leqslant p_{0}, \mathbb{P}$-вынуждающее $s_{0} \subset \boldsymbol{\xi}$. По выбору $\left\langle p_{0}, q_{0}\right\rangle$ можно предполагать, что

$$
\left\langle p^{\prime}, q^{\prime}\right\rangle(\mathbb{P} \times \mathbb{P}) \text {-вынуждает }\left\langle i_{0}, j_{0}, s\right\rangle \in \gamma\left(\boldsymbol{\xi}_{\text {пр }}\right) \wedge s \subset \boldsymbol{\xi}_{\text {л }}
$$

для подходящих $s \in \mathbb{S}$ и $q^{\prime} \in \mathbb{P}, q^{\prime} \leqslant q$. Это означает, что:

1) $p^{\prime} \mathbb{P}$-вынуждает $s \subset \boldsymbol{\xi}$;

2) $q^{\prime} \mathbb{P}$-вынуждает $\left\langle i_{0}, j_{0}, s\right\rangle \in \gamma(\boldsymbol{\xi})$.

В частности, согласно сказанному выше $p^{\prime}$ вынуждает $s_{0} \subset \boldsymbol{\xi}$ и $s \subset \boldsymbol{\xi}$, значит, либо $s \subseteq s_{0}$ - тогда пусть $s^{\prime}=s_{0}$, либо $s_{0} \subset s$ - тогда пусть $s^{\prime}=s$. В обоих случаях $\left\langle s^{\prime}, q^{\prime}\right\rangle(\mathbb{S} \times \mathbb{P})$-вынуждает $\left\langle i_{0}, j_{0}, s\right\rangle \in \gamma(\boldsymbol{\xi})$ и $s \subset \mathbf{a}$. Противоречие с (5). Лемма доказана.

Заметим, что $\mathbb{S}$ - подфорсинг форсинга Коэна $\mathbb{C}=\mathbb{N}<\omega$, значит, согласно лемме 25 сушествует $\mathbb{C}$-терм $\boldsymbol{\sigma}$ такой, что $\left\langle\Lambda, q_{0}\right\rangle(\mathbb{C} \times \mathbb{P})$-вьнуждает $\boldsymbol{\sigma} \in \vartheta^{\sharp}(\boldsymbol{\xi})$, следовательно, вынуждает $\boldsymbol{\sigma} \mathbb{E}^{\sharp} \boldsymbol{\xi}$. Рассматривая форсинг $\mathbb{C} \times \mathbb{P} \times \mathbb{P}$, вьводим, что, вообще, $\mathbb{C} \times \mathbb{P}$ вынуждает $\boldsymbol{\sigma} \mathbb{E}^{\sharp} \boldsymbol{\xi}$. Отсюда следует, что, во-первых, $\mathbb{C} \times \mathbb{C}$ вынужда-

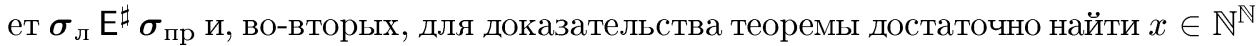
в $\mathbb{V}$ такой, что $\mathbb{C}$ вынуждает $x \mathbb{E}^{\sharp} \sigma$. Этим мы и займемся.

Через а обозначим $\mathbb{C}$-имя генерической по Коэну точки $\mathbb{N}^{\mathbb{N}}$. Терм $\boldsymbol{\sigma}$ может иметь сложную природу, однако можно его заменить термом вида $f^{\sharp}(\mathbf{a})$, где $f: \mathbb{N}^{\mathbb{N}} \rightarrow$ $\mathbb{N}^{\mathbb{N}}$ - борелевская функция в исходном универсуме $\mathbb{V}$. Из сказанного вьше вытекает, что для любой $(\mathbb{C} \times \mathbb{C})$-генерической над $\mathbb{V}$ пары $\langle\mathbf{a}, \mathbf{b}\rangle \in \mathbb{N}^{\mathbb{N}} \times \mathbb{N}^{\mathbb{N}}$ имеем $f^{\sharp}(\mathbf{a}) E^{\sharp} f^{\sharp}(\mathbf{b})$. Отсюда следует, что $f^{\sharp}(\mathbf{a}) E^{\sharp} f^{\sharp}(\mathbf{b})$ выполнено даже для любой пары отдельно генерических по Коэну $\mathbf{a}, \mathbf{b} \in \mathbb{N}^{\mathbb{N}}$. Таким образом, в том генерическом расширении $\mathbb{V}$, где множество всех генерических по Коэну точек котощее, найдется

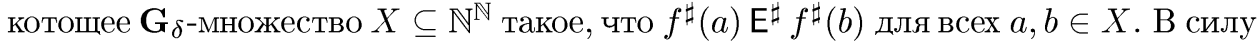
теоремы абсолютности Шенфилда утверждение о существовании такого множества $X$ истинно также и в $\mathbb{V}$, значит, в $\mathbb{V}$ имеется точка $x \in \mathbb{N}^{\mathbb{N}}$ такая, что $x \mathrm{E} f(a)$ выполнено для котощего множества точек $a \in \mathbb{N}^{\mathbb{N}}$. Это опять абсолютное по Шенфилду свойство $x$, значит, $\mathbb{C}$ вынуждает $x \mathrm{E}^{\sharp} f^{\sharp}(\mathbf{a})$, что и требовалось доказать.

Теорема 24 доказана.

4.6. Одно семейство приколотых идеалов. Назовем борелевский идеал $\mathscr{I}$ приколотым. если таково индуцированное ОЭ $\mathrm{E}_{\mathscr{I}}$. Из теоремы 22 следует, что все $P$-идеалы приколоты, ибо борелевские $P$-идеалы все "полишабельны" [15], а "польские" абелевы группы являются CLI-группами. Однако сушествуют и не "полишабельные" приколотые идеалы. 
Представим здесь одно семейство таких идеалов. Предположим, что $\left\{\varphi_{i}\right\}_{i \in \mathbb{N}}-$ семейство полунепрерывных снизу (l.s. с.) субмер на $\mathbb{N}$. Пусть

$$
\operatorname{Exh}_{\left\{\varphi_{i}\right\}}=\left\{X \subseteq \mathbb{N}: \varphi_{\infty}(X)=0\right\}, \quad \varphi_{\infty}(X)=\limsup _{i \rightarrow \infty} \varphi_{i}(X)
$$

- истощающий идеал последовательности субмер. По теореме Солецкого [15] для каждого борелевского $P$-идеала $\mathscr{I}$ сушествует l.s.c.-субмера $\varphi$ такая, что $\mathscr{I}=$ $\operatorname{Exh}_{\left\{\varphi_{i}\right\}}=\operatorname{Exh}_{\varphi}$, где $\varphi_{i}(x)=\varphi(x \cap[i, \infty))$, однако, например, не "полишабельный” идеал $\mathscr{I}_{1}=$ Fin $\times 0$ также имеет вид $\operatorname{Exh}_{\left\{\varphi_{i}\right\}}$, где для $x \subseteq \mathbb{N}^{2}$ определяется $\varphi_{i}(x)=0$ или $\varphi_{i}(x)=1$, когда $x \subseteq\{0, \ldots, n-1\} \times \mathbb{N}$ или $x \nsubseteq\{0, \ldots, n-1\} \times \mathbb{N}$ соответственно.

Теорема 26. Все идеаль вида $\operatorname{Exh}_{\left\{\varphi_{i}\right\}}$ приколоты.

ДоказАтельство. Пусть $\mathscr{I}=\operatorname{Exh}_{\left\{\varphi_{i}\right\}}$, где все $\varphi_{i}-$ l.s.c.-субмеры на $\mathbb{N}$. Можно предполагать, что субмеры $\varphi_{i}$ убывают, т. е. $\varphi_{i+1}(x) \leqslant \varphi_{i}(x)$ для каждого $x$, поскольку если это не так, то рассмотрим l. s. с.-субмеры $\varphi_{i}^{\prime}(x)=\sup _{j \geqslant i} \varphi_{j}(x)$.

Допустим, что $\mathrm{E}=\mathrm{E}_{\mathscr{I}}$ не приколото. Тогда существуют форсинг $\mathbb{P}$, виртуальный Е-класс $\boldsymbol{\xi}$ и “условие" $p \in \mathbb{P}$, которое $\mathbb{P}$-вынуждает $\neg x \mathrm{E}^{\sharp} \boldsymbol{\xi}$ для каждого $x \in$ $\mathscr{P}(\mathbb{N})$ в $\mathbb{V}$. По определению для всех $p^{\prime} \in \mathbb{P}$ и $n \in \mathbb{N}$ сушествуют $i \geqslant n$ и "условия" $q, r \in \mathbb{P}, q, r \leqslant p^{\prime}$, такие, что $\langle q, r\rangle(\mathbb{P} \times \mathbb{P})$-вынуждает неравенство $\varphi_{i}\left(\boldsymbol{\xi}_{\text {л }} \Delta \boldsymbol{\xi}_{\text {пр }}\right) \leqslant$ $2^{-n-1}$, следовательно, $\langle q, q\rangle(\mathbb{P} \times \mathbb{P})$-вынуждает $\varphi_{i}\left(\boldsymbol{\xi}_{\text {л }} \Delta \boldsymbol{\xi}_{\text {пр }}\right) \leqslant 2^{-n}$. Заключаем, что в $\mathbb{V}$ существуют последовательность чисел $i_{0}<i_{1}<i_{2}<\cdots$, последовательность $p_{0} \geqslant p_{1} \geqslant p_{2} \geqslant \cdots$ "условий" из $\mathbb{P}$ и множество $u_{n} \subseteq[0, n)$ для каждого $n$ такие, что $p_{0} \leqslant p$ и

1) каждое $p_{n} \mathbb{P}$-вынуждает $\boldsymbol{\xi} \cap[0, n)=u_{n}$;

$2)$ каждое $\left\langle p_{n}, p_{n}\right\rangle(\mathbb{P} \times \mathbb{P})$-вынуждает $\varphi_{i_{n}}\left(\boldsymbol{\xi}_{\text {л }} \Delta \boldsymbol{\xi}_{\text {пр }}\right) \leqslant 2^{-n}$.

В универсуме $\mathbb{V}$ положим $a=\bigcup_{n} u_{n}$; тогда $a \cap[0, n)=u_{n}$ для всех $n$. Мы утверждаем, что $p_{0}$ вынуждает $a \mathrm{E}^{\sharp} \boldsymbol{\xi}$. Это противоречит сделанному выше предположению, что доказывает теорему.

В самом деле, в противном случае найдется "условие" $q_{0} \leqslant p_{0}$, вынуждающее $\neg a \mathrm{E}^{\sharp} \boldsymbol{\xi}$. Рассмотрим генерическое расширение $\mathbb{V}^{+}$универсума, где существует последовательность $\mathbb{P}$-генерических множеств $U_{n} \subseteq \mathbb{P}$ такая, что для каждого $n$ пара $\left\langle U_{n}, U_{n+1}\right\rangle(\mathbb{P} \times \mathbb{P})$-генерична над $\mathbb{V}, p_{n} \in U_{n}$, и, дополнительно, $q_{0} \in U_{0}$. Тогда в $\mathbb{V}^{+}$множества $x_{n}=\boldsymbol{\xi}\left[U_{n}\right] \in \mathscr{P}(\mathbb{N})$ удовлетворяют $\varphi_{i_{n}}\left(x_{n} \Delta x_{m}\right) \leqslant 2^{-n}$ в силу 2$)$ всякий раз, когда $n \leqslant m$. Отсюда следует $\varphi_{i_{n}}\left(x_{n} \Delta a\right) \leqslant 2^{-n}$, поскольку $a=\lim _{m} x_{m}$ в силу условия 1$)$. Однако мы предположили, что субмеры $\varphi_{j}$ убывают, значит, $\varphi_{\infty}\left(x_{n} \Delta a\right) \leqslant 2^{-n}$. С другой стороны, $\varphi_{\infty}\left(x_{n} \Delta x_{0}\right)=0$, так как $\boldsymbol{\xi}-$ виртуальный Е-класс. Заключаем, что $\varphi_{\infty}\left(x_{0} \Delta a\right) \leqslant 2^{-n}$ для каждого $n$, другими словами, $\varphi_{\infty}\left(x_{0} \Delta a\right)=0$, т. е. $x_{0} \mathrm{E}^{\sharp} a$, что противоречит выбору $U_{0}$, поскольку $x_{0}=\boldsymbol{\xi}\left[U_{0}\right]$ и $q_{0} \in U_{0}$.

Вопрос 1. Являются ли приколотыми все борелевские идеалы? Ожидаемый положительный ответ показал бы, что $\mathrm{T}_{2}$ борелевски не сводится ни к какому борелевскому идеалу. Являются ли приколотыми все ОЭ, индуцированные борелевскими действиями борелевских CLI-групп? 
Вопрос 2 (Кехрис). Сушествует ли $\leqslant_{\mathrm{B}}$-наименьшее не приколотое борелевское ОЭ? Предполагалось, что $\mathrm{T}_{2}$ может быть таковым, однако Хъёрт информировал авторов о том, что существует строго $\leqslant{ }_{\mathrm{B}}$-меньшее не приколотое борелевское $\mathrm{O}$ достаточно сложной природы.

Авторы благодарны Г. Хъёрту, А. С. Кехрису и Су Гао за полезные обсуждения, относящиеся к содержанию этой статьи. Авторы благодарны Г. Хъёрту также за любезное разрешение включить в текст статьи один его неопубликованный результат (теорема 24).

\section{Список литературы}

1. Becker H., Kechris A.S. The descriptive set theory of Polish group actions. Cambridge: Cambridge University Press, 1996.

2. Farah I. Analytic quotients: theory of liftings for quotients over analytic ideals on the integers // Mem. Amer. Math. Soc. 2000. V. 148(702).

3. Friedman H., Stanley L. A Borel reducibility theory for classes of countable structures // J. Symbolic Logic. 1989. V. 54. № 3. P. 894-914.

4. Friedman H. Borel and Baire reducibility // Fund. Math. 2000. V. 164. № 1. P. 61-69.

5. Hjorth $G$. Orbit cardinals: on the effective cardinalities arising as quotient spaces of the form $X / G$ where $G$ acts on a Polish space $X / /$ Israel J. Math. 1999. V. 111. P. 221-261.

6. Hjorth $G$. Classification and orbit equivalence relations. Providence: American Math. Society, 2000.

7. Hjorth G., Kechris A.S. New dichotomies for Borel equivalence relations // Bull. Symbolic Logic. 1997. V. 3. № 3. P. 329-346.

8. Jackson S., Kechris A.S., Louveau A. Countable Borel equivalence relations // J. Math. Logic. 2002. V. 2. № 1. P. 1-80.

9. Kanovei $V$. An Ulm-type classification theorem for equivalence relations in Solovay model // J. Symbolic Logic. 1997. V. 62. № 4. P. 1333-1351.

10. Kanovei $V$. Ulm classification of analytic equivalence relations in generic universes // Math. Logic Quart. 1998. V. 44. № 3. P. 287-303.

11. Kechris A.S. Rigidity properties of Borel ideals on the integers // Topology Appl. 1998. V. 85. № 1-3. P. 195-205.

12. Kechris A.S. New directions in descriptive set theory // Bull. Symbolic Logic. 1999. V. 5. № 2. P. 161-174.

13. Kechris A.S. Actions of Polish groups and classification problems // Analysis and Logic. London Mathematical Society Lecture Note Series. Cambridge University Press, 2002. P. 168-237.

14. Louveau A. A separation theorem for $\Sigma_{1}^{1}$ sets // Trans. Amer. Math. Soc. 1980. V. 260. P. 363-378.

15. Solecki S. Analytic ideals and their applications // Ann. Pure Appl. Logic. 1999. V. 99. № 3. P. 51-72.

E-mail :

kanovei@math.uni-wuppertal.de,

reeken@math.uni-wuppertal.de
Поступило в редакцию

30.VII.2001 